\title{
Eigenvalue problems on domains with cracks II
}

\section{By Kazushi Yoshitomi}

(Received July 23, 2002)

(Communicated by Journal of Mathematical Sciences, the University of Tokyo)

\section{Introduction}

In this paper we investigate the eigenvalues of the Laplace operator on a region with a growing crack.

Let $\Omega$ be a bounded domain in $\mathbf{R}^{2}$ whose boundary is a smooth closed Jordan curve and let $\gamma:\left[0, t_{0}\right] \rightarrow \mathbf{R}^{2}$ be a smooth curve without self-intersection. We assume that

$$
\text { (A.1) } \gamma\left(\left(0, t_{0}\right)\right) \subset \Omega, \quad \gamma(0)=0 \in \partial \Omega, \quad \text { and } \quad \gamma\left(t_{0}\right) \in \partial \Omega
$$

For $\epsilon \in\left[0, t_{0}\right)$, we put

$$
\Omega_{\epsilon}=\Omega \backslash \gamma\left(\left[\epsilon, t_{0}\right)\right)
$$

Let

$$
0<\alpha<\pi
$$

For $b>0$, we define

$$
\Pi_{\alpha}^{b}=\left\{\left(x_{1}, x_{2}\right) \in \mathbf{R}^{2} ; x_{2}>0\right\} \backslash\left\{(r \cos \alpha, r \sin \alpha) \in \mathbf{R}^{2} ; r \geq b\right\} .
$$

For $a \in \mathbf{R}^{2}$ and $r>0$, we denote by $D(a, r)$ the open planar disk of radius $r$ centered at $\{a\}$. We impose the following assumptions on $\Omega$ and $\gamma$.

(A.2) There exists $r_{0} \in\left(0, t_{0}\right)$ such that

$$
\Omega_{\epsilon} \cap D\left(0, r_{0}\right)=\Pi_{\alpha}^{\epsilon} \cap D\left(0, r_{0}\right) \text { for all } \epsilon \in\left[0, r_{0}\right] \text {. }
$$

The set $\Omega_{0}$ consists of two connected components. Let $\Omega_{+}$and $\Omega_{-}$be the connected components of $\Omega_{0}$ which satisfy $\left(r_{0} / 2,0\right) \in \partial \Omega_{+}$and $\left(-r_{0} / 2,0\right) \in \partial \Omega_{-}$, respectively. We define

$$
Q_{\epsilon}=\text { the closure of }\left\{u \in C^{\infty}\left(\Omega_{\epsilon}\right) \cap H^{1}\left(\Omega_{\epsilon}\right) ; \operatorname{supp} u \cap \partial \Omega=\emptyset\right\} \text { in } H^{1}\left(\Omega_{\epsilon}\right),
$$

$Q^{ \pm}=$the closure of $\left\{u \in C^{\infty}\left(\Omega_{ \pm}\right) \cap H^{1}\left(\Omega_{ \pm}\right) ; \operatorname{supp} u \cap \partial \Omega_{ \pm} \cap \partial \Omega=\emptyset\right\}$ in $H^{1}\left(\Omega_{ \pm}\right)$ 


$$
\begin{aligned}
q_{\epsilon}(u, v) & =(\nabla u, \nabla v)_{L^{2}(\Omega)} \quad \text { for } u, v \in Q_{\epsilon}, \\
q^{ \pm}(u, v) & =(\nabla u, \nabla v)_{L^{2}\left(\Omega_{ \pm}\right)} \quad \text { for } \quad u, v \in Q^{ \pm} .
\end{aligned}
$$

Let $L_{\epsilon}$ be the self-adjoint operator associated with the quadratic form $q_{\epsilon}$. The operator $L_{\epsilon}$ is the negative Laplacian in $L^{2}\left(\Omega_{\epsilon}\right)$ subject to the Dirichlet boundary condition on $\partial \Omega$ and the Neumann boundary condition on the crack $\gamma\left(\left(\epsilon, t_{0}\right)\right)$. By $\lambda_{j}(\epsilon)$ we denote the $j$ th eigenvalue of $L_{\epsilon}$ counted with multiplicity. The aim of this paper is to find the full asymptotic expansions of the first two eigenvalues of $L_{\epsilon}$ as $\epsilon$ tends to zero. Let $L^{+}$and $L^{-}$be the self-adjoint operators associated with the quadratic forms $q^{+}$and $q^{-}$, respectively. The operator $L^{ \pm}$is the negative Laplacian in $L^{2}\left(\Omega_{ \pm}\right)$with the Dirichlet boundary condition on $\partial \Omega_{ \pm} \cap \partial \Omega$ and the Neumann boundary condition on $\gamma\left(\left(0, t_{0}\right)\right)$. Let $\lambda_{1}^{ \pm}<\lambda_{2}^{ \pm} \leq \lambda_{3}^{ \pm} \leq \cdots$ be the eigenvalues of $L^{ \pm}$ repeated according to multiplicity. Let $\Psi_{0}^{ \pm}(x)$ be the eigenvector of $L^{ \pm}$associated with the first eigenvalue $\lambda_{1}^{ \pm}$which is normalized by the conditions

$$
\Psi_{0}^{ \pm}(x)>0 \quad \text { in } \Omega_{ \pm}, \quad\left\|\Psi_{0}^{ \pm}\right\|_{L^{2}\left(\Omega_{ \pm}\right)}=1
$$

We put

$$
\beta=\frac{\alpha}{\pi}
$$

The functions $\Psi_{0}^{+}(x)$ and $\Psi_{0}^{-}(x)$ admit the following asymptotic expansions which can be differentiated term by term arbitrarily many times.

$$
\begin{gathered}
\Psi_{0}^{+}(x) \sim \sum_{j=1}^{\infty} \sum_{k=0}^{\infty} C_{j, k}^{+} r^{\frac{2 j-1}{2 \beta}+2 k} \sin \frac{(2 j-1) \theta}{2 \beta} \text { as } r \rightarrow 0, \\
C_{1,0}^{+}>0, \\
\Psi_{0}^{-}(x) \sim \sum_{j=1}^{\infty} \sum_{k=0}^{\infty} C_{j, k}^{-} r^{\frac{2 j-1}{2(1-\beta)}+2 k} \sin \frac{(2 j-1)(\pi-\theta)}{2(1-\beta)} \text { as } r \rightarrow 0, \\
C_{1,0}^{-}>0,
\end{gathered}
$$

where $(r, \theta)$ stand for the polar coordinates of $x$. We define

$$
\Gamma=\left\{\frac{a}{b} ;(a, b) \in \mathbf{N}^{2}, a \equiv 1 \quad(\bmod 2), b \equiv 0 \quad(\bmod 2)\right\} .
$$

Our main results are the following four theorems.

Theorem 0.1. Assume that $\beta \notin \Gamma$ and $\lambda_{1}^{+}<\lambda_{1}^{-}$. Then the eigenvalue $\lambda_{1}(\epsilon)$ admits the asymptotic expansion of the form

$$
\lambda_{1}(\epsilon) \sim \lambda_{1}^{+}+\sum_{m=1}^{\infty} \sum_{n=0}^{\infty} \sum_{p=0}^{\infty} \lambda_{m, n, p} \epsilon^{\frac{m}{\beta}+\frac{n}{1-\beta}+2 p} \quad \text { as } \epsilon \rightarrow 0
$$


where

$$
\lambda_{1,0,0}=\frac{\pi}{4}(1-\beta)^{\frac{1-\beta}{\beta}}\left(C_{1,0}^{+}\right)^{2} .
$$

Theorem 0.2. Assume that $\beta \notin \Gamma, \lambda_{1}^{+}=\lambda_{1}^{-}$, and $\alpha>\pi / 2$. Then the eigenvalues $\lambda_{1}(\epsilon)$ and $\lambda_{2}(\epsilon)$ admit the asymptotic expansions of the form

$$
\begin{array}{ll}
\lambda_{1}(\epsilon) \sim \lambda_{1}^{+}+\sum_{m=1}^{\infty} \sum_{n=0}^{\infty} \sum_{p=0}^{\infty} \lambda_{m, n, p}^{-} \epsilon^{\frac{m}{1-\beta}+\frac{n}{\beta}+2 p} & \text { as } \epsilon \rightarrow 0 \\
\lambda_{2}(\epsilon) \sim \lambda_{1}^{+}+\sum_{m=1}^{\infty} \sum_{n=0}^{\infty} \sum_{p=0}^{\infty} \lambda_{m, n, p}^{+} \epsilon^{\frac{m}{\beta}+\frac{n}{1-\beta}+2 p} & \text { as } \epsilon \rightarrow 0
\end{array}
$$

where $\lambda_{1,0,0}^{+}$is equal to the right side of $(0.8)$ and $\lambda_{1,0,0}^{-}$is given by the formula

$$
\lambda_{1,0,0}^{-}=\frac{\pi}{4} \beta^{\frac{\beta}{1-\beta}}\left(C_{1,0}^{-}\right)^{2} .
$$

Theorem 0.3. Assume that $\beta \in \Gamma$ and $\lambda_{1}^{+}<\lambda_{1}^{-}$. Let $(q, s)$ be a unique pair of natural numbers such that

$$
\beta=\frac{q}{s}, \quad \operatorname{gcd}(q, s)=1 .
$$

Then the eigenvalue $\lambda_{1}(\epsilon)$ admits the asymptotic expansion of the form

$$
\lambda_{1}(\epsilon) \sim \lambda_{1}^{+}+\sum_{m=1}^{\infty} \sum_{n=0}^{\infty} \sum_{p=0}^{\infty} \sum_{h=0}^{\infty} \lambda_{m, n, p, h} \epsilon^{\frac{m}{\beta}+\frac{n}{1-\beta}+2 p}\left(\epsilon^{s} \log \epsilon\right)^{h} \quad \text { as } \quad \epsilon \rightarrow 0
$$

where $\lambda_{1,0,0,0}$ equals the right side of $(0.8)$.

Theorem 0.4. Assume that $\beta \in \Gamma, \lambda_{1}^{+}=\lambda_{1}^{-}$, and $\alpha>\pi / 2$. Let $(q, s)$ be a unique pair of natural numbers such that

$$
\beta=\frac{q}{s}, \quad \operatorname{gcd}(q, s)=1 .
$$

Then the eigenvalues $\lambda_{1}(\epsilon)$ and $\lambda_{2}(\epsilon)$ admit the asymptotic expansions of the form

$$
\begin{aligned}
& \lambda_{1}(\epsilon) \sim \lambda_{1}^{+}+\sum_{m=1}^{\infty} \sum_{n=0}^{\infty} \sum_{p=0}^{\infty} \sum_{h=0}^{\infty} \lambda_{m, n, p, h}^{-} \epsilon^{\frac{m}{1-\beta}+\frac{n}{\beta}+2 p}\left(\epsilon^{s} \log \epsilon\right)^{h} \quad \text { as } \epsilon \rightarrow 0, \\
& \lambda_{2}(\epsilon) \sim \lambda_{1}^{+}+\sum_{m=1}^{\infty} \sum_{n=0}^{\infty} \sum_{p=0}^{\infty} \sum_{h=0}^{\infty} \lambda_{m, n, p, h}^{+} \epsilon^{\frac{m}{\beta}+\frac{n}{1-\beta}+2 p}\left(\epsilon^{s} \log \epsilon\right)^{h} \quad \text { as } \epsilon \rightarrow 0,
\end{aligned}
$$

where $\lambda_{1,0,0,0}^{+}$and $\lambda_{1,0,0,0}^{-}$are given by the right sides of (0.8) and (0.9), respectively. 
Our study is inspired and motivated by the work of M. Dauge and B. Helffer. By using the method of variation, they proved in [2] that

$$
\lim _{\epsilon \rightarrow 0} \lambda_{j}(\epsilon)=\nu_{j} \quad \text { for } j \in \mathbf{N}
$$

where $\nu_{1} \leq \nu_{2} \leq \cdots$ are the rearrangement of $\left\{\lambda_{j}^{+}\right\}_{j=1}^{\infty} \cup\left\{\lambda_{j}^{-}\right\}_{j=1}^{\infty}$ counted with multiplicity. This result drew our interest in the asymptotic behavior of $\lambda_{j}(\epsilon)$ as $\epsilon$ tends to zero. In our previous work [9] the full asymptotic expansions of $\lambda_{1}(\epsilon)$ and $\lambda_{2}(\epsilon)$ are obtained in the case when $\alpha=\pi / 2$ and $\lambda_{1}^{+}=\lambda_{1}^{-}$. In the derivation of these asymptotic expansions, we made use of a reflection argument in the vicinity of the origin. The scope of this paper is to obtain the full asymptotic expansions of the eigenvalues of $L_{\epsilon}$ as $\epsilon$ tends to zero in the case when $\alpha \neq \pi / 2$. In the proofs of Theorems $0.1-0.4$ we need a tool which differs from the reflection argument used in [9], because the region $\Omega_{\epsilon}$ has no reflection symmetry in a neighborhood of the origin.

We prove the main theorems by using the method of matched asymptotic expansion (see [5] and [3]). We define

$$
\xi=\epsilon^{-1} x .
$$

In the proof of Theorem 0.1, we look for the approximate first eigenvalue of $L_{\epsilon}$ and the associated approximate eigenvector in the following form.

$$
\begin{aligned}
\lambda(\epsilon) & =\lambda_{1}^{+}+\sum_{m=1}^{\infty} \sum_{n=0}^{\infty} \sum_{p=0}^{\infty} \lambda_{m, n, p} \epsilon^{\frac{m}{\beta}+\frac{n}{1-\beta}+2 p} \\
\Psi_{\epsilon}^{\text {out },+}(x) & =\Psi_{0}^{+}(x)+\sum_{j=1}^{\infty} \sum_{k=0}^{\infty} \sum_{l=0}^{\infty} \epsilon^{\frac{j}{\beta}+\frac{k}{1-\beta}+2 l} \Psi_{j, k, l}^{+}(x) \quad \text { in } \Omega_{+} \backslash D(0, \sqrt{\epsilon}), \\
\Psi_{\epsilon}^{\text {out },-}(x) & =\sum_{j=1}^{\infty} \sum_{k=1}^{\infty} \sum_{l=0}^{\infty} \epsilon^{\frac{2 j-1}{2 \beta}+\frac{2 k-1}{2(1-\beta)}+2 l} \Psi_{j, k, l}^{-}(x) \quad \text { in } \Omega_{-} \backslash D(0, \sqrt{\epsilon}) \\
\Psi_{\epsilon}^{\text {in }}(x) & =\sum_{j=1}^{\infty} \sum_{k=0}^{\infty} \sum_{l=0}^{\infty} \epsilon^{\frac{2 j-1}{2 \beta}+\frac{k}{1-\beta}+2 l} v_{j, k, l}(\xi) \quad \text { in } \Omega_{\epsilon} \cap D(0,2 \sqrt{\epsilon}) .
\end{aligned}
$$

Inserting (0.11) and (0.12) into the equation $\left(\Delta_{x}+\lambda(\epsilon)\right) \Psi_{\epsilon}^{\text {out },+}(x)=0$ and identifying the powers of $\epsilon$, we have

$$
\begin{gathered}
\left(\Delta_{x}+\lambda_{1}^{+}\right) \Psi_{j, k, l}^{+}(x)=-\lambda_{j, k, l} \Psi_{0}^{+}(x)-\sum_{m=1}^{j-1} \sum_{n=0}^{k} \sum_{p=0}^{l} \lambda_{m, n, p} \Psi_{j-m, k-n, l-p}^{+}(x) \text { in } \Omega_{+}, \\
\Psi_{j, k, l}^{+}(x)=0 \quad \text { on } \partial \Omega_{+} \cap \partial \Omega, \quad \frac{\partial}{\partial n} \Psi_{j, k, l}^{+}(x)=0 \quad \text { on } \gamma\left(\left(0, t_{0}\right)\right) .
\end{gathered}
$$


In a similar way, we obtain the following equations from (0.11) and (0.13).

$$
\begin{gathered}
\left(\Delta_{x}+\lambda_{1}^{+}\right) \Psi_{j, k, l}^{-}(x)=-\sum_{m=1}^{j-1} \sum_{n=0}^{k-1} \sum_{p=0}^{l} \lambda_{m, n, p} \Psi_{j-m, k-n, l-p}^{-}(x) \text { in } \Omega_{-}, \\
\Psi_{j, k, l}^{-}(x)=0 \quad \text { on } \partial \Omega_{-} \cap \partial \Omega, \quad \frac{\partial}{\partial n} \Psi_{j, k, l}^{-}(x)=0 \quad \text { on } \gamma\left(\left(0, t_{0}\right)\right) .
\end{gathered}
$$

Plugging (0.11) and (0.14) into the equation $\left(\Delta_{x}+\lambda(\epsilon)\right) \Psi_{\epsilon}^{\text {in }}(x)=0$ and equating the powers of $\epsilon$ on account of $\Delta_{x}=\epsilon^{-2} \Delta_{\xi}$, we get

$$
\begin{gathered}
\Delta_{\xi} v_{j, k, l}(\xi)=-\lambda_{1}^{+} v_{j, k, l-1}(\xi)-\sum_{m=1}^{j-1} \sum_{n=0}^{k} \sum_{p=0}^{l-1} \lambda_{m, n, p} v_{j-m, k-n, l-p-1}(\xi) \text { in } \Pi_{\alpha}^{1}, \\
v_{j, k, l}=0 \quad \text { on } \Upsilon_{1}, \quad \frac{\partial}{\partial n_{ \pm}} v_{j, k, l}(\xi)=0 \quad \text { for } \xi \in \Upsilon_{2, \alpha},
\end{gathered}
$$

where

$$
\begin{gathered}
\Upsilon_{1}:=\mathbf{R} \times\{0\}, \quad \Upsilon_{2, \alpha}:=\partial \Pi_{\alpha}^{1} \backslash(\mathbf{R} \times\{0\}) \\
\frac{\partial}{\partial n_{ \pm}} v_{j, k, l}(\xi):=\lim _{h \rightarrow \pm 0} \frac{v_{j, k, l}\left(\xi+h n_{0}\right)-v_{j, k, l}(\xi)}{h} \text { for } \xi \in \Upsilon_{2, \alpha}
\end{gathered}
$$

and $n_{0}=(\sin \alpha,-\cos \alpha)$ is the unit normal vector to $\Upsilon_{2, \alpha}$. We shall construct the formal power series $\Psi_{\epsilon}^{\text {out },+}(x), \Psi_{\epsilon}^{\text {out },-}(x)$, and $\Psi_{\epsilon}^{\text {in }}(x)$ in such a way that $\Psi_{\epsilon}^{\text {in }}(x)$ asymptotically coincides with $\Psi_{\epsilon}^{\text {out },+}(x)$ and $\Psi_{\epsilon}^{\text {out },-}(x)$ on the intermediate regions $\Omega_{+} \cap(D(0,2 \sqrt{\epsilon}) \backslash D(0, \sqrt{\epsilon}))$ and $\Omega_{-} \cap(D(0,2 \sqrt{\epsilon}) \backslash D(0, \sqrt{\epsilon}))$, respectively.

We organize this paper as follows. Because the proofs of Theorems 0.1-0.4 are similar, we give a detailed proof of Theorem 0.1 in Sections 1-3, and we show Theorems 0.2-0.4 with omitting details in Sections 4 and 5. In Section 1, we solve the outer equations $(0.15)_{j, k, l}$ and $(0.16)_{j, k, l}$. We also analyze the asymptotic behavior of the solutions to the equations $(0.15)_{j, k, l}$ and $(0.16)_{j, k, l}$ in a neighborhood of the origin. For this purpose we use the $L^{2}$-theory of elliptic differential equations on coner domains which was originated by V.A. Kondrat'ev [6]. In Section 2, we solve the inner equation $(0.17)_{j, k, l}$. We give an explicit formula for the solution to this equation. In the construction of this formula and the analysis of the asymptotic expansion of the solution in a neighborhood of infinity, a conformal map plays the most important role. Moreover the map enables us to derive the explicit formulae $(0.8)$ and (0.9). This map is one of the most significant tools in the proofs of the main theorems. In Section 3 we construct the coefficients of $(0.11)-(0.14)$ by combining an induction procedure with the results in the previous sections. In the construction we need matching conditions which ensures the mentioned coincidence of the expansions (0.12)-(0.14) on the intermediate regions (see (ii) in Theorem 3.1). 
In Section 4, we give a proof of Theorem 0.2. In section 5 , we prove Theorems 0.3 and 0.4 by using approximate solutions which are derived by adding logarithmic terms to (0.11)-(0.14). In Section 6, we compare the main theorems with the results of our previous work [9].

Throughout this paper we adopt the following conventions to reduce complicated classifications. We denote inessential constants by $C$ on estimations. For $k, l \in \mathbf{Z}$ with $k>l$, we define $\sum_{j=k}^{l} a_{j}=0$ and $\left\{b_{j}\right\}_{k \leq j \leq l}=\emptyset$. A formula which contains either \pm or $\mp$ means two formulae which correspond to the upper sign and the lower sign, respectively. For example, the formula $a^{ \pm}=b^{\mp}$ means that $a^{+}=b^{-}$ and $a^{-}=b^{+}$. We also regard undefined terms as zero in formulae. For example, if $a$ and $c$ are defined and if $b$ is undefined, then the formula $a+b=c$ means $a=c$.

\section{Outer equations}

In order to solve the outer equations $(0.15)_{j, k, l}$ and $(0.16)_{j, k, l}$, we use the $L^{2}$ theory of elliptic differential equations on domains with conic singularities which was inspired by V.A. Kondrat'ev [6]. For $\mu \in(0,2 \pi)$, we put

$$
\mathbf{K}_{\mu}=\left\{(r \cos \theta, r \sin \theta) \in \mathbf{R}^{2} ; r>0,0<\theta<\mu\right\} .
$$

Let $\mathbf{R}_{+}$be the set of all positive real numbers. By $(r, \theta) \in \mathbf{R}_{+} \times(0, \mu)$ we denote the polar coordinates of $x \in \mathbf{K}_{\mu}$. We look at the equation

$$
\left\{\begin{aligned}
-\Delta_{x} u(r, \theta) & =f(r, \theta) \quad \text { in } \mathbf{K}_{\mu} \\
u(\cdot, 0) & =0 \quad \text { on } \mathbf{R}_{+} \\
\frac{\partial}{\partial \theta} u(\cdot, \mu) & =0 \text { on } \mathbf{R}_{+} .
\end{aligned}\right.
$$

By $\mathbf{Z}_{+}$we denote the set of all non-negative integers. For $l \in \mathbf{Z}_{+}$and $\tau \in \mathbf{R}$, we define

$$
V_{\tau}^{l}\left(\mathbf{K}_{\mu}\right)=\left\{u \in \mathcal{D}^{\prime}\left(\mathbf{K}_{\mu}\right) ; r^{\tau-l+|\delta|} \partial_{x}^{\delta} u(x) \in L^{2}\left(\mathbf{K}_{\mu}\right) \text { for } \delta \in \mathbf{Z}_{+}^{2},|\delta| \leq l\right\},
$$

where $\mathcal{D}^{\prime}\left(\mathbf{K}_{\mu}\right)$ stands for the space of all distributions on $\mathbf{K}_{\mu}$. Let

$$
T_{\mu}=\left\{\frac{\pi}{2 \mu}(2 j-1) ; j \in \mathbf{Z}\right\}
$$

From [6] we recall the following two theorems (see also [7, Chapter 2]).

Theorem 1.1 (V.A. Kondrat'ev). Let $l \in \mathbf{Z}_{+}$and $\tau \in \mathbf{R}$. Assume that $\tau-l-1 \notin T_{\mu}$ and $f \in V_{\tau}^{l}\left(\mathbf{K}_{\mu}\right)$. Then the equation (1.1) has a unique solution in $V_{\tau}^{l+2}\left(\mathbf{K}_{\mu}\right)$. 
Theorem 1.2 (V.A. Kondrat'ev). Let $l \in \mathbf{Z}_{+}, \tau_{1}<\tau_{2}, \tau_{k}-l-1 \notin T_{\mu}$ for $k=1,2$, and $f \in V_{\tau_{1}}^{l}\left(\mathbf{K}_{\mu}\right) \cap V_{\tau_{2}}^{l}\left(\mathbf{K}_{\mu}\right)$. For $k=1,2$, let $u_{k} \in V_{\tau_{k}}^{l+2}\left(\mathbf{K}_{\mu}\right)$ be the unique solution of (1.1). Then we have

$$
u_{1}(x)-u_{2}(x)=\sum_{n \in \mathcal{A}\left(\tau_{1}, \tau_{2}, l\right)} c_{n} r^{\frac{\pi}{2 \mu}(2 n-1)} \sin \frac{(2 n-1) \pi}{2 \mu} \theta \text { in } \mathbf{K}_{\mu}
$$

where

$$
\mathcal{A}\left(\tau_{1}, \tau_{2}, l\right)=\left\{n \in \mathbf{Z} ; \quad l+1-\tau_{2}<\frac{\pi}{2 \mu}(2 n-1)<l+1-\tau_{1}\right\}
$$

Now we introduce function spaces which we need in the sequel. For $j \in \mathbf{N}$, we define

$$
\begin{aligned}
S^{j}\left(\mathbf{K}_{\mu}\right) & :=\bigcap_{l=0}^{\infty} V_{l+1}^{l+2 j}\left(\mathbf{K}_{\mu}\right) \\
& =\left\{u \in \mathcal{D}^{\prime}\left(\mathbf{K}_{\mu}\right) ; r^{-2 j+1+|\delta|} \partial_{x}^{\delta} u \in L^{2}\left(\mathbf{K}_{\mu}\right) \text { for all } \delta \in \mathbf{Z}_{+}^{2}\right\} .
\end{aligned}
$$

For an open set $\Sigma$ in $\mathbf{R}^{2}$, a finite subset $S$ of $\partial \Sigma$, and $k \in \mathbf{N} \cup\{\infty\}$, we define

$$
\begin{array}{r}
C^{k}(\bar{\Sigma} \backslash S)=\left\{u: \Sigma \rightarrow \mathbf{R} ; u \in C^{k}(\overline{\Sigma \backslash A}) \quad \text { for any open set } A\right. \text { such that } \\
S \subset A \text { and } \Sigma \backslash A \neq \emptyset\} .
\end{array}
$$

Choose $\chi \in C^{\infty}([0, \infty))$ such that

$$
\chi(r)=1 \quad \text { on }\left[0, r_{0} / 4\right], \quad \chi(r)=0 \quad \text { on }\left[r_{0} / 2, \infty\right)
$$

For $m \in \mathbf{Z}_{+}$, we define $J_{m}^{+}$as the class of functions $u$ defined on $\Omega_{+}$which satisfy the conditions (a), (b), and (c) below.

(a) $u \in C^{\infty}\left(\overline{\Omega_{+}} \backslash\left\{0, \gamma\left(t_{0}\right)\right\}\right),(1-\chi(r)) u \in L^{2}\left(\Omega_{+}\right)$.

(b) $u=0$ on $\partial \Omega_{+} \cap \partial \Omega, \frac{\partial}{\partial n} u=0$ on $\gamma\left(\left(0, t_{0}\right)\right)$.

(c) The function $u$ admits the asymptotic expansion of the form

$u(x) \sim \sum_{j=0}^{\infty} \sum_{k=0}^{\infty} D_{j, k} r^{\frac{2 j-2 m+1}{2 \beta}+2 k} \sin \frac{(2 j-2 m+1) \theta}{2 \beta} \quad$ as $r \rightarrow 0, \quad x \in \Omega_{+}$,

which can be differentiated term by term infinitely many times.

For $f, g \in \cap_{r \in\left(0, r_{0}\right)} L^{2}\left(\Omega_{ \pm} \backslash D(0, r)\right)$, we define

$$
(f, g)_{\Omega_{ \pm}}=\lim _{r \rightarrow+0}(f, g)_{L^{2}\left(\Omega_{ \pm} \backslash D(0, r)\right)}
$$

if and only if the limit exists. 
In this section our main aim is to prove the following lemma.

LeMmA 1.3. Let $\beta \notin \Gamma, m \in \mathbf{Z}_{+}$, and $f \in J_{m}^{+}$. Given $\left\{a_{j}\right\}_{j=0}^{m} \subset \mathbf{R}$, there exists $\mu \in \mathbf{R}$ such that the equation

$$
\left\{\begin{aligned}
\left(\Delta+\lambda_{1}^{+}\right) \varphi & =-\mu \Psi_{0}^{+}+f \quad \text { in } \Omega_{+}, \\
\varphi & =0 \quad \text { on } \partial \Omega_{+} \cap \partial \Omega, \\
\frac{\partial}{\partial n} \varphi & =0 \quad \text { on } \gamma\left(\left(0, t_{0}\right)\right), \\
\left(\varphi, \Psi_{0}^{+}\right)_{\Omega_{+}} & =0
\end{aligned}\right.
$$

has a solution $\varphi \in J_{m+1}^{+}$which admits the asymptotic expansion

$$
\varphi(x) \sim \sum_{j=0}^{\infty} \sum_{k=0}^{\infty} E_{j, k} r^{\frac{2 j-2 m-1}{2 \beta}+2 k} \sin \frac{(2 j-2 m-1) \theta}{2 \beta} \quad \text { as } r \rightarrow 0, \quad x \in \Omega_{+}
$$

with

$$
E_{j, 0}=a_{j} \text { for } 0 \leq j \leq m .
$$

In order to prove this Lemma, we need the asymptotic representation (0.3) of the function $\Psi_{0}^{+}$. Supposing this fact for a moment, we shall complete the proof of this Lemma.

Proof of Lemma 1.3. Since $f \in J_{m}^{+}$, the function $f$ admits the asymptotic expansion

$$
f(x) \sim \sum_{j=0}^{\infty} \sum_{k=0}^{\infty} D_{j, k} r^{\frac{2 j-2 m+1}{2 \beta}+2 k} \sin \frac{(2 j-2 m+1) \theta}{2 \beta} \text { as } r \rightarrow 0 .
$$

Let $F$ be the partial sum of the formal power series on the right side of (1.5):

$$
F(x)=\sum_{j=0}^{m-1} \sum_{k=0}^{\infty} D_{j, k} r^{\frac{2 j-2 m+1}{2 \beta}+2 k} \sin \frac{(2 j-2 m+1) \theta}{2 \beta} .
$$

Thanks to the condition $\beta \notin \Gamma$ and the formula

$$
\Delta_{x}\left(r^{\frac{2 j-1}{2 \beta}+2 k} \sin \frac{(2 j-1) \theta}{2 \beta}\right)=2 k\left(\frac{2 j-1}{\beta}+2 k\right) r^{\frac{2 j-1}{2 \beta}+2 k-2} \sin \frac{(2 j-1) \theta}{2 \beta},
$$

we can construct the formal power series

$$
\Psi(x)=\sum_{j=0}^{m} \sum_{k=0}^{\infty} E_{j, k} r^{\frac{2 j-2 m-1}{2 \beta}+2 k} \sin \frac{(2 j-2 m-1) \theta}{2 \beta}
$$


satisfying $\left(\Delta+\lambda_{1}^{+}\right) \Psi=F$ term by term and

$$
E_{j, 0}=a_{j} \text { for } 0 \leq j \leq m \text {. }
$$

For $p \in \mathbf{R}$, we put $[p]=\max \{l \in \mathbf{Z} ; l \leq p\}$. We define

$$
M(j, N)=\left[\frac{N+m-j+1}{2 \beta}\right]+1 \quad \text { for } N, j \in \mathbf{Z}_{+} .
$$

Then we have

$$
\frac{2 j-2 m-1}{2 \beta}+2 M(j, N)>\frac{2 N+1}{2 \beta} .
$$

We introduce the following partial sum of $\Psi$ :

$$
\Psi^{N}(x):=\sum_{j=0}^{m} \sum_{k=0}^{M(j, N)} E_{j, k} r^{\frac{2 j-2 m-1}{2 \beta}+2 k} \sin \frac{(2 j-2 m-1) \theta}{2 \beta}
$$

We seek the solution of (1.2) which admits the form

$$
\varphi(x)=\chi(r) \Psi^{N}(x)+\phi_{N}(x), \quad \phi_{N} \in \operatorname{Dom}\left(L_{+}\right) .
$$

Inserting this into the equation (1.2), we obtain the equation for $\phi_{N}$ :

$$
\left\{\begin{aligned}
\left(\Delta+\lambda_{1}^{+}\right) \phi_{N} & =-\mu \Psi_{0}^{+}+g_{N} \quad \text { in } \Omega_{+} \\
\phi_{N} & =0 \text { on } \partial \Omega_{+} \cap \partial \Omega \\
\frac{\partial}{\partial n} \phi_{N} & =0 \text { on } \gamma\left(\left(0, t_{0}\right)\right) \\
\left(\phi_{N}, \Psi_{0}^{+}\right)_{L^{2}\left(\Omega_{+}\right)} & =-\left(\chi(r) \Psi^{N}(x), \Psi_{0}^{+}\right)_{\Omega_{+}}
\end{aligned}\right.
$$

where

$$
\begin{aligned}
g_{N}=(1-\chi) f-\Psi^{N} \Delta \chi-2 \nabla \chi \cdot \nabla \Psi^{N} \\
+\chi\left(f-\sum_{j=0}^{m-1} \sum_{k=0}^{M(j+1, N)-1} D_{j, k} r^{\frac{2 j-2 m+1}{2 \beta}+2 k} \sin \frac{(2 j-2 m+1) \theta}{2 \beta}\right. \\
\left.\quad-\lambda_{1}^{+} \sum_{j=0}^{m} E_{j, M(j, N)} r^{\frac{2 j-2 m-1}{2 \beta}+2 M(j, N)} \sin \frac{(2 j-2 m-1) \theta}{2 \beta}\right) .
\end{aligned}
$$

From (1.5) and (1.9), we have $-\mu \Psi_{0}^{+}+g_{N} \in L^{2}\left(\Omega_{+}\right)$. Since $\lambda_{1}^{+}$is a simple eigenvalue of $L_{+}$, we infer that the equation (1.11) has a solution in $\operatorname{Dom}\left(L_{+}\right)$ if and only if $\left(-\mu \Psi_{0}^{+}+g_{N}, \Psi_{0}^{+}\right)_{L^{2}\left(\Omega_{+}\right)}=0$; i.e. $\mu=\left(g_{N}, \Psi_{0}^{+}\right)_{L^{2}\left(\Omega_{+}\right)}$. We define 
$\mu_{N}=\left(g_{N}, \Psi_{0}^{+}\right)_{L^{2}\left(\Omega_{+}\right)}$. For $\mu=\mu_{N}$, let $\phi_{N} \in \operatorname{Dom}\left(L_{+}\right)$be the unique solution of the equation (1.11). We put $\varphi_{N}(x)=\chi(r) \Psi^{N}(x)+\phi_{N}(x)$.

Let us show that $\mu_{N}$ and $\varphi_{N}$ are independent of the choice of $N \in \mathbf{Z}_{+}$. For $N, M \in \mathbf{Z}_{+}$, we have

$$
\begin{gathered}
\left(\Delta+\lambda_{1}^{+}\right)\left(\varphi_{N}-\varphi_{M}\right)=\left(\mu_{N}-\mu_{M}\right) \Psi_{0}^{+} \quad \text { in } \Omega_{+}, \\
\left(\varphi_{N}-\varphi_{M}, \Psi_{0}^{+}\right)_{\Omega_{+}}=0 \\
\varphi_{N}(x)-\varphi_{M}(x)=\chi(r)\left(\Psi^{N}(x)-\Psi^{M}(x)\right)+\phi_{N}(x)-\phi_{M}(x) \in \operatorname{Dom}\left(L_{+}\right) .
\end{gathered}
$$

Since $\lambda_{1}^{+}$is a simple eigenvalue of $L_{+}$, we get $\mu_{N}-\mu_{M}=0$ and $\varphi_{N}-\varphi_{M}=0$. Thus $\mu_{N}$ and $\varphi_{N}$ are independent of the choice of $N \in \mathbf{Z}_{+}$, which we denote by $\mu$ and $\varphi$, respectively.

Our next task is to prove that $\varphi \in J_{m+1}^{+}$. For $n \in \mathbf{Z}_{+}$, we define

$$
l(n)=\left[\frac{(2 n-1) \pi}{4 \alpha}\right]+1
$$

Since $\beta \notin \Gamma$, we get

$$
2(l(n)-1)<\frac{(2 n-1) \pi}{2 \alpha}<2 l(n)
$$

Let us demonstrate the following claim.

ClaIm. For any $n \in \mathbf{Z}_{+}$, the function $\chi \phi_{n}$ admits the representation

$$
\chi \phi_{n}=\chi\left(\sum_{j=m+1}^{m+n} \sum_{k=0}^{M(j, n)} A_{j, k} r^{\frac{2 j-2 m-1}{2 \beta}+2 k} \sin \frac{(2 j-2 m-1) \theta}{2 \beta}\right)+w_{n}
$$

where $w_{n} \in S^{l(n+1)}\left(\mathbf{K}_{\alpha}\right)$ and

$$
\begin{aligned}
& \Delta\left(A_{j, k} r^{\frac{2 j-2 m-1}{2 \beta}+2 k} \sin \frac{(2 j-2 m-1) \theta}{2 \beta}\right) \\
& =\left(-\lambda_{1}^{+} A_{j, k-1}-\mu C_{j-m, k-1}^{+}+D_{j-1, k-1}\right) r^{\frac{2 j-2 m-1}{2 \beta}+2 k-2} \sin \frac{(2 j-2 m-1) \theta}{2 \beta}
\end{aligned}
$$

for $m+1 \leq j \leq m+n, 1 \leq k \leq M(j, n)$.

We prove this Claim by induction on $n$. Let us show that $(1.13)_{n}$ holds for $n=0$. As a preliminary, we first prove that $\chi \phi_{0} \in S^{1}\left(\mathbf{K}_{\alpha}\right)$ by induction. Since $\phi_{0} \in \operatorname{Dom}\left(L_{+}\right)$, we have the Hardy type inequality

$$
\int_{\Omega_{+} \cap D\left(0, r_{0}\right)}\left|\nabla_{x} \phi_{0}\right|^{2} d x \geq \int_{\Omega_{+} \cap D\left(0, r_{0}\right)} r^{-2}\left|\partial_{\theta} \phi_{0}\right|^{2} d x
$$




$$
\begin{aligned}
& =\int_{0}^{\alpha} \int_{0}^{r_{0}} r^{-1}\left|\partial_{\theta} \phi_{0}\right|^{2} d r d \theta \\
& \geq \frac{\pi^{2}}{4 \alpha^{2}} \int_{0}^{\alpha} \int_{0}^{r_{0}} r^{-1}\left|\phi_{0}\right|^{2} d r d \theta \\
& =\frac{\pi^{2}}{4 \alpha^{2}} \int_{\Omega_{+} \cap D\left(0, r_{0}\right)} r^{-2}\left|\phi_{0}\right|^{2} d x .
\end{aligned}
$$

So we get $\chi \phi_{0} \in V_{0}^{1}\left(\mathbf{K}_{\alpha}\right)$. Now we assume that $\chi \phi_{0} \in V_{k}^{k+1}\left(\mathbf{K}_{\alpha}\right)$ for some $k \in \mathbf{Z}_{+}$. For $N \in \mathbf{Z}_{+}$, we obtain

$$
\left\{\begin{aligned}
& \Delta\left(\chi \phi_{N}\right)=\chi(\left.-\lambda_{1}^{+} \phi_{N}-\mu \Psi_{0}^{+}+g_{N}\right) \\
&+2 \nabla \chi \cdot \nabla \phi_{N}+\phi_{N} \Delta \chi=: h_{N} \text { in } \mathbf{K}_{\alpha} \\
&\left(\chi \phi_{N}\right)(\cdot, 0)=0 \quad \text { on } \mathbf{R}_{+} \\
& \frac{\partial}{\partial \theta}\left(\chi \phi_{N}\right)(\cdot, \alpha)=0 \quad \text { on } \mathbf{R}_{+} .
\end{aligned}\right.
$$

Since $h_{0} \in V_{k+1}^{k}\left(\mathbf{K}_{\alpha}\right)$, we infer from Theorem 1.1 that there exists $v \in V_{k+1}^{k+2}\left(\mathbf{K}_{\alpha}\right)$ such that

$$
\left\{\begin{aligned}
\Delta v & =h_{0} & & \text { in } \mathbf{K}_{\alpha} \\
v(\cdot, 0) & =0 & & \text { on } \mathbf{R}_{+} \\
\frac{\partial}{\partial \theta} v(\cdot, \alpha) & =0 & & \text { on } \mathbf{R}_{+} .
\end{aligned}\right.
$$

Let us prove that $v=\chi \phi_{0}$. Pick $\chi_{0} \in C^{\infty}([0, \infty))$ such that $\chi_{0}(r)=1$ on $[0,1]$ and $\chi_{0}(r)=0$ on $[2, \infty)$. We put $p_{R, \delta}(x)=\left(1-\chi_{0}(r / \delta)\right) \chi_{0}(r / R)\left(\chi \phi_{0}-v\right)$ for $R, \delta>0$. We get $\int_{\mathbf{K}_{\alpha}}\left|\nabla p_{R, \delta}(x)\right|^{2} d x=-\int_{\mathbf{K}_{\alpha}} p_{R, \delta} \Delta p_{R, \delta} d x$. Letting $R \rightarrow \infty$ and $\delta \rightarrow 0$ on this equality by noting $\Delta\left(\chi \phi_{0}-v\right)=0$ and $\chi \phi_{0}-v \in V_{0}^{1}\left(\mathbf{K}_{\alpha}\right)$, we have $\int_{\mathbf{K}_{\alpha}}\left|\nabla\left(\chi \phi_{0}-v\right)\right|^{2} d x=0$ and $v=\chi \phi_{0} \in V_{k+1}^{k+2}\left(\mathbf{K}_{\alpha}\right)$. Thus we obtain $\chi \phi_{0} \in V_{k}^{k+1}\left(\mathbf{K}_{\alpha}\right)$ for all $k \in \mathbf{Z}_{+}$and hence $\chi \phi_{0} \in S^{1}\left(\mathbf{K}_{\alpha}\right)$.

Next we prove by induction that $\chi \phi_{0} \in S^{j}\left(\mathbf{K}_{\alpha}\right)$ for $j \leq l(1)$. Let $1 \leq k<l(1)$ and assume that $\chi \phi_{0} \in S^{k}\left(\mathbf{K}_{\alpha}\right)$. Since $\chi \phi_{0} \in S^{0}\left(\mathbf{K}_{\alpha}\right) \cap S^{k}\left(\mathbf{K}_{\alpha}\right)$, we have $h_{0} \in$ $S^{0}\left(\mathbf{K}_{\alpha}\right) \cap S^{k}\left(\mathbf{K}_{\alpha}\right)$. Combining this with Theorem 1.2, (1.14) $)_{0}$, and the fact that $-\frac{\pi}{2 \alpha}<0<2 k<\frac{\pi}{2 \alpha}$, we obtain $\chi \phi_{0} \in S^{k+1}\left(\mathbf{K}_{\alpha}\right)$. Hence we have $\chi \phi_{0} \in S^{l(1)}\left(\mathbf{K}_{\alpha}\right)$.

Assume that $(1.13)_{n}$ is valid for some $n \in \mathbf{Z}_{+}$. Inserting $(1.13)_{n}$ into $(1.14)_{n}$, we obtain the equation for $w_{n}$ :

$$
\left\{\begin{aligned}
\Delta w_{n} & =-\lambda_{1}^{+} w_{n}+\tilde{h}_{n} \quad \text { in } \mathbf{K}_{\alpha} \\
w_{n}(\cdot, 0) & =0 \text { on } \mathbf{R}_{+} \\
\frac{\partial}{\partial \theta} w_{n}(\cdot, \alpha) & =0 \text { on } \mathbf{R}_{+}
\end{aligned}\right.
$$


where

$$
\begin{aligned}
\tilde{h}_{n}= & -\lambda_{1}^{+} \chi \sum_{j=m+1}^{m+n} A_{j, M(j, n)} r^{\frac{2 j-2 m-1}{2 \beta}+2 M(j, n)} \sin \frac{(2 j-2 m-1) \theta}{2 \beta} \\
& -\mu \chi\left(\Psi_{0}^{+}-\sum_{j=1}^{n} \sum_{k=0}^{M(j+m, n)-1} C_{j, k}^{+} r^{\frac{2 j-1}{2 \beta}+2 k} \sin \frac{(2 j-1) \theta}{2 \beta}\right) \\
& +\chi\left(g_{n}-\sum_{j=m}^{n+m-1} \sum_{k=0}^{M(j+1, n)-1} D_{j, k} r^{\frac{2 j-2 m+1}{2 \beta}+2 k} \sin \frac{(2 j-2 m+1) \theta}{2 \beta}\right) \\
& +2 \nabla \chi \cdot \nabla \phi_{n}+\phi_{n} \Delta \chi \\
& -(\Delta \chi) \sum_{j=m+1}^{m+n} \sum_{k=0}^{M(j, n)} A_{j, k} r^{\frac{2 j-2 m-1}{2 \beta}+2 k} \sin \frac{(2 j-2 m-1) \theta}{2 \beta} \\
& -2 \nabla \chi \cdot \nabla\left(\sum_{j=m+1}^{m+n} \sum_{k=0}^{M(j, n)} A_{j, k} r^{\frac{2 j-2 m-1}{2 \beta}+2 k} \sin \frac{(2 j-2 m-1) \theta}{2 \beta}\right) .
\end{aligned}
$$

Using (1.9) and (1.12), we have $\tilde{h}_{n} \in S^{l(n+1)}\left(\mathbf{K}_{\alpha}\right)$. So we get

$$
-\lambda_{1}^{+} w_{n}+\tilde{h}_{n} \in S^{l(n+1)-1}\left(\mathbf{K}_{\alpha}\right) \cap S^{l(n+1)}\left(\mathbf{K}_{\alpha}\right) \text {. }
$$

Combining this with (1.15) and Theorem 1.2, we infer that $w_{n}$ admits the representation

$$
w_{n}=c_{n} r^{\frac{2 n+1}{2 \beta}} \sin \frac{(2 n+1) \theta}{2 \beta}+q_{n}, \quad q_{n} \in S^{l(n+1)+1}\left(\mathbf{K}_{\alpha}\right)
$$

We introduce the following partial sum of the asymptotic expansion of $\tilde{h}_{n}(x)$ as $r \rightarrow 0$.

$$
\begin{aligned}
H_{n}= & -\lambda_{1}^{+} \sum_{j=m+1}^{m+n} A_{j, M(j, n)} r^{\frac{2 j-2 m-1}{2 \beta}+2 M(j, n)} \sin \frac{(2 j-2 m-1) \theta}{2 \beta} \\
& -\mu\left(\sum_{j=1}^{n} \sum_{k=M(j+m, n)}^{\infty}+\sum_{j=n+1}^{n+1} \sum_{k=0}^{\infty}\right) C_{j, k}^{+} r^{\frac{2 j-1}{2 \beta}+2 k} \sin \frac{(2 j-1) \theta}{2 \beta} \\
& -\lambda_{1}^{+} \sum_{j=0}^{m} E_{j, M(j, n)} r^{\frac{2 j-2 m-1}{2 \beta}+2 M(j, n)} \sin \frac{(2 j-2 m-1) \theta}{2 \beta} \\
& +\left(\sum_{j=0}^{m-1} \sum_{k=M(j+1, n)}^{\infty}+\sum_{j=m}^{n+m-1} \sum_{k=M(j+1, n)}^{\infty}+\sum_{j=n+m}^{n+m} \sum_{k=0}^{\infty}\right) D_{j, k} \\
& \times r^{\frac{2 j-2 m+1}{2 \beta}+2 k} \sin \frac{(2 j-2 m+1) \theta}{2 \beta} .
\end{aligned}
$$


Let

$$
\begin{aligned}
G= & \sum_{k=0}^{\infty} g_{n, k} r^{\frac{2 n+1}{2 \beta}+2 k} \sin \frac{(2 n+1) \theta}{2 \beta} \\
& +\sum_{j=0}^{m+n} \sum_{k=M(j, n)+1}^{\infty} B_{j, k} r^{\frac{2 j-2 m-1}{2 \beta}+2 k} \sin \frac{(2 j-2 m-1) \theta}{2 \beta}
\end{aligned}
$$

be the formal power series satisfying

$$
g_{n, 0}=c_{n}
$$

and

$$
\left(\Delta+\lambda_{1}^{+}\right) G=H_{n} .
$$

By the construction of the formal power series $G$ and $\Psi$, we have

$$
B_{j, k}=E_{j, k} \quad \text { for } \quad 0 \leq j \leq m, \quad k \geq M(j, n)+1 .
$$

We further introduce the following partial sum of $G$.

$$
\begin{aligned}
\tilde{G}= & \sum_{k=0}^{M(m+n+1, n+1)} g_{n, k} r^{\frac{2 n+1}{2 \beta}+2 k} \sin \frac{(2 n+1) \theta}{2 \beta} \\
& +\sum_{j=0}^{m+n} \sum_{k=M(j, n)+1}^{M(j, n+1)} B_{j, k} r^{\frac{2 j-2 m-1}{2 \beta}+2 k} \sin \frac{(2 j-2 m-1) \theta}{2 \beta}
\end{aligned}
$$

We put

$$
\tilde{q}_{n}=w_{n}-\chi \tilde{G}
$$

From (1.16) and (1.18), we have $\tilde{q}_{n} \in S^{l(n+1)+1}\left(\mathbf{K}_{\alpha}\right)$. Inserting $w_{n}=\tilde{q}_{n}+\chi \tilde{G}$ into the equation (1.15), we obtain

$$
\begin{aligned}
\Delta \tilde{q}_{n} & =-\lambda_{1}^{+} \tilde{q}_{n}+\tilde{h}_{n}-\chi\left(\Delta+\lambda_{1}^{+}\right) \tilde{G}-2 \nabla \chi \cdot \nabla \tilde{G}-\tilde{G} \Delta \chi \\
& =:-\lambda_{1}^{+} \tilde{q}_{n}+k_{n} .
\end{aligned}
$$

From (1.9), (1.12), and (1.19), we have $k_{n} \in S^{l(n+2)}\left(\mathbf{K}_{\alpha}\right)$. By induction, let us show that $\tilde{q}_{n} \in S^{j}\left(\mathbf{K}_{\alpha}\right)$ for $l(n+1)+1 \leq j \leq l(n+2)$. Assume that $\tilde{q}_{n} \in S^{k}\left(\mathbf{K}_{\alpha}\right)$ for some $l(n+1)+1 \leq k \leq l(n+2)-1$. Since $-\lambda_{1}^{+} \tilde{q}_{n}+k_{n} \in S^{k-1}\left(\mathbf{K}_{\alpha}\right) \cap S^{k}\left(\mathbf{K}_{\alpha}\right)$ and since $\frac{(2 n+1) \pi}{2 \alpha}<2(k-1)<2 k<\frac{(2 n+3) \pi}{2 \alpha}$, we obtain $\tilde{q}_{n} \in S^{k+1}\left(\mathbf{K}_{\alpha}\right)$ by using Theorem 1.2 and (1.22). Thus we get $\tilde{q}_{n} \in S^{l(n+2)}\left(\mathbf{K}_{\alpha}\right)$. Notice that

$$
\begin{aligned}
\chi \phi_{n+1} & =\chi \phi_{n}+\chi^{2}\left(\Psi^{n}-\Psi^{n+1}\right) \\
& =\chi \phi_{n}+\chi\left(\Psi^{n}-\Psi^{n+1}\right)-\chi(1-\chi)\left(\Psi^{n}-\Psi^{n+1}\right)
\end{aligned}
$$


Combining this with $(1.13)_{n},(1.20)$, and $(1.21)$, we have

$$
\begin{aligned}
\chi \phi_{n+1}=\chi( & \sum_{j=m+1}^{m+n} \sum_{k=0}^{M(j, n)} A_{j, k} r^{\frac{2 j-2 m-1}{2 \beta}+2 k} \sin \frac{(2 j-2 m-1) \theta}{2 \beta} \\
& +\sum_{k=0}^{M(m+n+1, n+1)} g_{n, k} r^{\frac{2 n+1}{2 \beta}+2 k} \sin \frac{(2 n+1) \theta}{2 \beta} \\
& \left.+\sum_{j=m+1}^{m+n} \sum_{k=M(j, n)+1}^{M(j, n+1)} B_{j, k} r^{\frac{2 j-2 m-1}{2 \beta}+2 k} \sin \frac{(2 j-2 m-1) \theta}{2 \beta}\right) \\
& +\tilde{q}_{n}-(1-\chi) \chi\left(\Psi^{n}-\Psi^{n+1}\right) .
\end{aligned}
$$

This together with the construction of $G$ implies that $(1.13)_{n+1}$ is valid. Hence we obtain the assertion of the Claim.

Using $w_{n} \in S^{l(n+1)}\left(\mathbf{K}_{\alpha}\right)$ and Sobolev's imbedding theorem, we get

$$
w_{n} \in C^{2 l(n+1)-3}\left(\overline{\mathbf{K}_{\alpha}}\right)
$$

and

$$
\left|\partial_{x}^{\delta} w_{n}\right| \leq C_{\delta} r^{2 l(n+1)-3-|\delta|} \quad \text { on } \quad \mathbf{K}_{\alpha} \quad \text { for } \delta \in \mathbf{Z}_{+}^{2}, \quad|\delta| \leq 2 l(n+1)-3 .
$$

This together with $(1.13)_{n}$ and (1.10) implies that $\varphi \in J_{m+1}^{+}$.

Proof of (0.3)-(0.6). We omit the proofs of $(0.3)$ and $(0.5)$ because they are easier than that of (1.3). In order to prove (0.4), we extend $\Psi_{0}^{+}(r, \theta)$ to the function $\tilde{\Psi}_{0}^{+}(r, \theta)$ on $W=\left\{(r, \theta) ; 0<r<r_{0}, 0<\theta<2 \alpha\right\}$ by the formula

$$
\tilde{\Psi}_{0}^{+}(r, \theta)= \begin{cases}\Psi_{0}^{+}(r, \theta) & \text { for } 0<\theta \leq \alpha \\ \Psi_{0}^{+}(r, 2 \alpha-\theta) & \text { for } \alpha \leq \theta<2 \alpha .\end{cases}
$$

Then we have $-\Delta \tilde{\Psi}_{0}^{+}=\lambda_{1}^{+} \tilde{\Psi}_{0}^{+}$on $W, \tilde{\Psi}_{0}^{+} \in H^{1}(W) \cap C^{\infty}(\bar{W} \backslash\{0\}), \tilde{\Psi}_{0}^{+}>0$ on $W, \tilde{\Psi}_{0}^{+}(\cdot, 0)=\tilde{\Psi}_{0}^{+}(\cdot, 2 \alpha)=0$ on $\left(0, r_{0}\right)$, and the asymptotic representation

$$
\tilde{\Psi}_{0}^{+}(r, \theta) \sim \sum_{j=1}^{\infty} \sum_{k=0}^{\infty} C_{j, k}^{+} r^{\frac{2 j-1}{2 \beta}+2 k} \sin \frac{(2 j-1) \theta}{2 \beta} \quad \text { as } r \rightarrow 0, \quad(r, \theta) \in W .
$$

By mimicking the proof of $[1$, Proposition 19.2], we obtain (0.4). Similarly we get (0.6).

Next we look at the other outer equation. For $m \in \mathbf{Z}_{+}$, we define $J_{m}^{-}$as the class of functions $u$ defined on $\Omega_{-}$which satisfy the conditions (d), (e), and (f) below. 
(d) $u \in C^{\infty}\left(\overline{\Omega_{-}} \backslash\left\{0, \gamma\left(t_{0}\right)\right\}\right),(1-\chi(r)) u \in L^{2}\left(\Omega_{-}\right)$.

(e) $u=0$ on $\partial \Omega_{-} \cap \partial \Omega, \frac{\partial}{\partial n} u=0$ on $\gamma\left(\left(0, t_{0}\right)\right)$.

(f) The function $u$ admits the asymptotic expansion of the form

$$
u(x) \sim \sum_{j=0}^{\infty} \sum_{k=0}^{\infty} D_{j, k} r^{\frac{2 j-2 m+1}{2(1-\beta)}+2 k} \sin \frac{(2 j-2 m+1)(\pi-\theta)}{2(1-\beta)} \quad \text { as } r \rightarrow 0, \quad x \in \Omega_{-},
$$

which can be differentiated term by term infinitely many times.

Acting as in the proof of Lemma 1.3, we have the following claim.

Lemma 1.4. Let $\beta \notin \Gamma, \lambda_{1}^{+}<\lambda_{1}^{-}, m \in \mathbf{Z}_{+}$, and $f \in J_{m}^{-}$. Given $\left\{a_{j}\right\}_{j=0}^{m-1} \subset$ $\mathbf{R}$, the equation

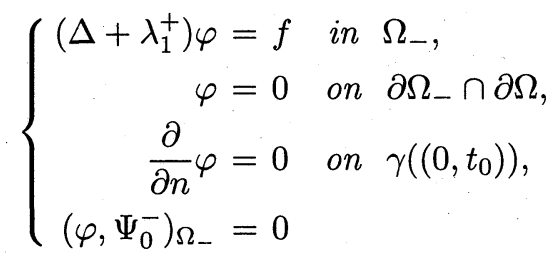

has a solution $\varphi \in J_{m}^{-}$which admits the asymptotic expansion

$$
\varphi(x) \sim \sum_{j=0}^{\infty} \sum_{k=0}^{\infty} D_{j, k} r^{\frac{2 j-2 m+1}{2(1-\beta)}+2 k} \sin \frac{(2 j-2 m+1)(\pi-\theta)}{2(1-\beta)} \quad \text { as } \quad r \rightarrow 0, \quad x \in \Omega_{-}
$$

with

$$
D_{j, 0}=a_{j} \quad \text { for } 0 \leq j \leq m-1
$$

\section{Inner equations}

Our first task in this section is to derive an explicit formula for the solution to the equation

$$
\left\{\begin{array}{l}
\Delta_{\xi} u(\xi)=f(\xi) \text { in } \Pi_{\alpha}^{1} \\
u=0 \text { on } \Upsilon_{1}, \quad \frac{\partial}{\partial n_{ \pm}} u(\xi)=0 \text { for } \xi \in \Upsilon_{2, \alpha}
\end{array}\right.
$$

For this purpose we use the techniques of conformal maps. We identify $\mathbf{R}^{2}$ with $\mathbf{C}$ by the map $\mathbf{R}^{2} \ni(x, y) \mapsto x+i y \in \mathbf{C}$. It is readily seen that the Green function of the equation

$$
\left\{\begin{array}{l}
\Delta_{z} u(z)=g(z) \text { in } Q:=\mathbf{R}_{+}+i \mathbf{R}_{+} \\
u=0 \text { on } \mathbf{R}_{+}, \quad \frac{\partial}{\partial z_{1}} u=0 \text { on } i \mathbf{R}_{+}
\end{array}\right.
$$


is given by the formula

$$
G(z, w)=\frac{1}{2 \pi} \log \frac{|z-w||z+\bar{w}|}{|z+w||z-\bar{w}|}
$$

Thus, it suffices to construct a conformal map $\Phi$ from $\Pi_{\alpha}^{1}$ onto $Q$ which maps $\Upsilon_{1}$ and $\Upsilon_{2, \alpha}$ onto $\mathbf{R}_{+}$and $i \mathbf{R}_{+}$, respectively.

We shall construct the conformal map $\Phi$ by composing some elementary conformal maps. We put

$$
P=\{z \in \mathbf{C} ; \operatorname{Im} z>0\} .
$$

We first introduce the Schwarz-Christoffel map $H: P \rightarrow \mathbf{C}$ by the formula

$$
\begin{aligned}
H(w) & =\beta(1-\beta) \int_{0}^{w} z(z-1)^{\beta-2} d z+e^{i \alpha} \\
& =(1-\beta)(w-1)^{\beta}-\beta(w-1)^{\beta-1}
\end{aligned}
$$

where $z^{t}=\exp (t \log z), \log z=\log |z|+i \arg z$, and $\arg z \in(-\pi, \pi]$ for $z \in \mathbf{C} \backslash\{0\}$. Let us demonstrate the following claim.

Proposition 2.1. The function $H$ is a conformal map from $P$ onto $\Pi_{\alpha}^{1}$. It sends $(-\infty, 0],[0,1)$, and $(1, \infty)$ onto $\Upsilon_{2, \alpha}, \Upsilon_{2, \alpha}$, and $\Upsilon_{1}$, respectively.

Proof. By the Schwarz-Christoffel theorem (see [8, Chapter V, Section 6]), we claim that $H$ is a conformal map from $P$ onto the (generalized) triangle with edges $H((-\infty, 0]), H([0,1))$, and $H((1, \infty))$. From $(2.2)$ we have $H((1, \infty))=\Upsilon_{1}$. It also follows from (2.2) that

$$
H(w)=e^{i \alpha}(1-w)^{\beta-1}(1-(1-\beta) w) \text { for } w \in(-\infty, 1) .
$$

Thus we obtain $H((-\infty, 0])=H([0,1))=\Upsilon_{2, \alpha}$. The proof is complete.

Next we define

$$
S(z)=(z-1)^{1 / 2}, \quad z \in P .
$$

The function $S$ is a conformal map from $P$ onto $Q$ which maps $(-\infty, 1)$ and $(1, \infty)$ onto $i \mathbf{R}_{+}$and $\mathbf{R}_{+}$, respectively. Finally, we define

$$
\Phi=S \circ H^{-1}
$$

Then the function $\Phi$ is a conformal map from $\Pi_{\alpha}^{1}$ onto $Q$ which maps $\Upsilon_{1}$ and $\Upsilon_{2, \alpha}$ onto $\mathbf{R}_{+}$and $i \mathbf{R}_{+}$, respectively. Thus the solution to the equation (2.1) is given by the formula

$$
u(\xi)=\int_{\Pi_{\alpha}^{1}} G(\Phi(\xi), \Phi(w)) f(w) d w, \quad \xi \in \Pi_{\alpha}^{1} .
$$


Thanks to this formula, we can get the asymptotic expansion of the solution to the equation (2.1). Let

$$
\kappa=\min \{1-\beta, \beta\}
$$

We define

$$
\begin{array}{r}
H_{\text {comp }}^{1}\left(\Pi_{\alpha}^{1}\right)=\left\{u: \Pi_{\alpha}^{1} \rightarrow \mathbf{R} ; u \in H^{1}(A) \text { for any bounded open set } A\right. \\
\text { such that } \left.\emptyset \neq A \subset \Pi_{\alpha}^{1}\right\}
\end{array}
$$

Proposition 2.2. Let $N \in \mathbf{R}$ and $N \geq 2+\frac{5}{\kappa}$. Assume that $f$ is bounded and locally Lipschitz continuous in $\Pi_{\alpha}^{1}$. We also suppose that $f$ satisfies the condition

$$
f(w)=\mathcal{O}\left(|w|^{-N}\right) \quad \text { as } \quad|w| \rightarrow \infty
$$

Then the function $u(\xi)$ from (2.4) admits the following asymptotic expansions as $|\xi| \rightarrow \infty$ which can be differentiated term by term once:

$$
\begin{gathered}
u(\xi)=\sum_{j=1}^{M} c_{j} \rho^{-\frac{2 j-1}{2 \beta}} \sin \frac{(2 j-1) \theta}{2 \beta}+\mathcal{O}\left(\rho^{-\frac{2 M+1}{2 \beta}}\right) \quad \text { for } 0<\theta<\alpha, \\
u(\xi)=\sum_{j=1}^{M} d_{j} \rho^{-\frac{2 j-1}{2(1-\beta)}} \sin \frac{(2 j-1)(\pi-\theta)}{2(1-\beta)}+\mathcal{O}\left(\rho^{-\frac{2 M+1}{2(1-\beta)}}\right) \quad \text { for } \alpha<\theta<\pi,
\end{gathered}
$$

where $(\rho, \theta) \in \mathbf{R}_{+} \times(0, \pi)$ are the polar coordinates of $\xi$ and $M=\left[\frac{\kappa(N-2)-1}{2}\right]-1(\geq$ 1). Moreover, we have $u \in H_{\text {comp }}^{1}\left(\Pi_{\alpha}^{1}\right) \cap C^{2}\left(\Pi_{\alpha}^{1}\right) \cap L^{\infty}\left(\Pi_{\alpha}^{1}\right),\left.u\right|_{\Lambda_{ \pm}} \in C^{1}\left(\overline{\Lambda_{ \pm}} \backslash\left\{e^{i \alpha}\right\}\right)$, and $\left|\xi-e^{i \alpha}\right|^{1 / 2} \nabla u(\xi) \in L^{\infty}\left(D\left(e^{i \alpha}, 1 / 2\right)\right)$.

Proof. The proof of this proposition is analogous to that of $[10$, Proposition 2.3]. We have

$$
2 \pi G(z, \zeta)=\operatorname{Re}\left\{\log \left(1-\frac{\zeta}{z}\right)+\log \left(1+\frac{\zeta}{\bar{z}}\right)-\log \left(1+\frac{\zeta}{z}\right)-\log \left(1-\frac{\zeta}{\bar{z}}\right)\right\}
$$

Since

$$
\left|\operatorname{Re}\left\{\log (1-t)+\sum_{j=1}^{n} \frac{t^{j}}{j}\right\}\right| \leq C_{n}|t|^{n+1} \quad \text { for } t \in \mathbf{C}, \quad|t-1| \geq \frac{1}{2}
$$

and since

$$
|t|^{j} \leq C_{j}|\log | 1-t|| \quad \text { for } t \in \mathbf{C}, \quad|t-1| \leq \frac{1}{2}
$$


we infer that the kernel $G(z, \zeta)$ admits the following expression for $m \in \mathbf{N}$ :

$$
\begin{gathered}
G(z, \zeta)=\sum_{j=1}^{m} \frac{2}{(2 j-1) \pi} \operatorname{Im}\left(z^{-2 j+1}\right) \operatorname{Im}\left(\zeta^{2 j-1}\right)+H_{m}(z, \zeta) \\
\left|H_{m}(z, \zeta)\right| \leq C_{m}^{\prime}|z|^{-2 m-1}|\zeta|^{2 m+1} \quad \text { for } \zeta \in R_{z}:=K_{z} \cap K_{-z} \cap K_{\bar{z}} \cap K_{-\bar{z}} \\
\left|H_{m}(z, \zeta)\right| \leq C_{m}^{\prime}\left\{|\log | 1-\frac{\zeta}{z}||+|\log | 1+\frac{\zeta}{\bar{z}}||+|\log | 1+\frac{\zeta}{z} \|+|\log | 1-\frac{\zeta}{\bar{z}}||\right\} \\
\text { for } \zeta \in R_{z}^{c},
\end{gathered}
$$

where

$$
K_{z}:=\left\{\zeta \in \mathbf{C} ;|z-\zeta| \geq \frac{1}{2}|z|\right\} .
$$

Applying the Schwarz reflection principle to $H^{-1}\left(-z^{\beta-1}\right)$ and $1 / H^{-1}\left(z^{-\beta}\right)$ on $D\left(0, \frac{1}{2}\right) \cap P$ and $D\left(0, \frac{1}{2}\right) \backslash \bar{P}$, respectively, we see that these functions extend to the holomorphic functions on $D\left(0, \frac{1}{2}\right)$. Thus $H^{-1}(z)$ admits the Puiseux series expansions with real coefficients for $|z|>2$ :

$$
\begin{aligned}
& H^{-1}(z)=\sum_{j=0}^{\infty} q_{j} z^{\frac{1-j}{\beta}} \quad \text { for } 0<\arg z<\alpha \\
& H^{-1}(z)=1+\sum_{j=1}^{\infty} p_{j}(-z)^{-\frac{j}{1-\beta}} \quad \text { for } \quad \alpha<\arg z<\pi .
\end{aligned}
$$

Combining these with (2.3), we claim that $\Phi$ admits the Puiseux series expansions

$$
\begin{aligned}
& \Phi(w)=\sum_{j=0}^{\infty} r_{j} w^{\frac{1-2 j}{2 \beta}} \text { for }|w|>2, \quad 0<\arg w<\alpha \\
& \Phi(w)=\sum_{j=0}^{\infty} s_{j} w^{\frac{-1-2 j}{2(1-\beta)}} \quad \text { for }|w|>2, \quad \alpha<\arg w<\pi .
\end{aligned}
$$

Let $0<\arg \xi<\alpha$ and $|\xi|>2$. We put $M=\left[\frac{\kappa(N-2)-1}{2}\right]-1$. Using (2.7), (2.9), and (2.10), we express the kernel $G(\Phi(\xi), \Phi(w))$ as follows.

$$
\begin{gathered}
G(\Phi(\xi), \Phi(w))=\sum_{j=1}^{M} K_{j}(w) \rho^{\frac{-2 j+1}{2 \beta}} \sin \frac{(2 j-1) \theta}{2 \beta}+L_{M}(\xi, w) \\
\left|K_{j}(w)\right| \leq C_{j}^{\prime \prime}(1+|w|)^{\frac{2 j-1}{2 \beta}}
\end{gathered}
$$




$$
\begin{array}{r}
\left|L_{M}(\xi, w)\right| \leq C_{M}^{\prime \prime}(1+|w|)^{\frac{2 M+1}{2 \beta}}|\xi|^{-\frac{2 M+1}{2 \beta}} \quad \text { for } w \in \Phi^{-1}\left(R_{\Phi(\xi)}\right), \\
\left|L_{M}(\xi, w)\right| \leq C_{M}^{\prime \prime}\left\{|\log | 1-\frac{\Phi(w)}{\Phi(\xi)}||+|\log | 1+\frac{\Phi(w)}{\overline{\Phi(\xi)}} \|\right. \\
\left.+|\log | 1+\frac{\Phi(w)}{\Phi(\xi)}||+|\log | 1-\frac{\Phi(w)}{\overline{\Phi(\xi)}}||\right\} \\
\text { for } w \in \Phi^{-1}\left(R_{\Phi(\xi)}^{c}\right) .
\end{array}
$$

From (2.4), we obtain

$$
\begin{aligned}
u(\xi)= & \sum_{j=1}^{M} c_{j} \rho^{\frac{-2 j+1}{2 \beta}} \sin \frac{(2 j-1) \theta}{2 \beta}+\int_{\Phi^{-1}\left(R_{\Phi(\xi)}\right)} L_{M}(\xi, w) f(w) d w \\
& +\int_{\Phi^{-1}\left(R_{\Phi(\xi)}^{c}\right)} L_{M}(\xi, w) f(w) d w
\end{aligned}
$$

where $c_{j}=\int_{\Pi_{\alpha}^{1}} K_{j}(w) f(w) d w$. It follows from (2.11) that

$$
\left|\int_{\Phi^{-1}\left(R_{\Phi(\xi)}\right)} L_{M}(\xi, w) f(w) d w\right| \leq C \rho^{-\frac{2 M+1}{2 \beta}} \int_{\Pi_{\alpha}^{1}}(1+|w|)^{\frac{2 M+1}{2 \beta}-N} d w .
$$

Since $\frac{2 M+1}{2 \beta}-N<-2$, we have $\int_{\Pi_{\alpha}^{1}}(1+|w|)^{\frac{2 M+1}{2 \beta}-N} d w<\infty$. From $(2.12)$, we get

$$
\begin{aligned}
& \left|\int_{\Phi^{-1}\left(R_{\Phi(\xi)}^{c}\right)} L_{M}(\xi, w) f(w) d w\right| \\
& \leq C \int_{R_{\Phi(\xi)}^{c}}\left\{|\log | 1-\frac{y}{\Phi(\xi)}\left\|+|\log | 1+\frac{y}{\overline{\Phi(\xi)}}\right\|+|\log | 1+\frac{y}{\Phi(\xi)} \|\right. \\
& \left.+|\log | 1-\frac{y}{\overline{\Phi(\xi)}} \|\right\}\left|f\left(\Phi^{-1}(y)\right)\right|\left|\left(\Phi^{-1}\right)^{\prime}(y)\right|^{2} d y .
\end{aligned}
$$

Since $R_{\Phi(\xi)}^{c} \subset\{\zeta \in \mathbf{C} ;|\zeta| \leq 2|\Phi(\xi)|\}$ and $|\Phi(\xi)| \leq C^{\prime}|\xi|^{\frac{1}{2 \beta}}$, we have

$$
\begin{aligned}
& \begin{array}{l}
\left|\int_{\Phi^{-1}\left(R_{\Phi(\xi)}^{c}\right)} L_{M}(\xi, w) f(w) d w\right| \\
\leq C \int_{D\left(0, C^{\prime} \rho^{\frac{1}{2 \beta}}\right)}\left\{|\log | 1-\frac{y}{\Phi(\xi)}||+|\log | 1+\frac{y}{\Phi(\xi)}||+|\log | 1+\frac{y}{\Phi(\xi)}||\right. \\
\left.+|\log | 1-\frac{y}{\overline{\Phi(\xi)}}||\right\}|y|^{-2 \beta N}|y|^{2(2 \beta-1)} d y \\
\leq C \rho^{-N+\frac{2 \beta-1}{\beta}} \int_{D\left(0, C^{\prime} \rho^{\frac{1}{2 \beta}}\right)}\{|\log | y-\Phi(\xi)||+|\log | \bar{y}+\Phi(\xi)||+|\log | y+\Phi(\xi)|| \\
\quad+|\log | \bar{y}-\Phi(\xi)||+4|\log | \Phi(\xi)||\} d y .
\end{array}
\end{aligned}
$$


Since $\int_{D\left(0, C^{\prime} \rho^{\frac{1}{2 \beta}}\right)}|\log | y|| d y=\mathcal{O}\left(\rho^{\frac{1}{\beta}} \log \rho\right)$, we get

$$
\left|\int_{\Phi^{-1}\left(R_{\Phi(\xi)}^{c}\right)} L_{M}(\xi, w) f(w) d w\right|=\mathcal{O}\left(\rho^{-N+2} \log \rho\right) .
$$

Noting again that $N-2>\frac{2 M+1}{2 \beta}$, we obtain

$$
u(\xi)=\sum_{j=1}^{M} c_{j} \rho^{-\frac{2 j-1}{2 \beta}} \sin \frac{(2 j-1) \theta}{2 \beta}+\mathcal{O}\left(\rho^{-\frac{2 M+1}{2 \beta}}\right) \quad \text { for } \quad \arg \xi \in(0, \alpha) .
$$

Applying a similar method to the derivatives of $u$, we arrive at (2.5). The proof of (2.6) is similar to that of $(2.5)$.

From (2.4) we have

$$
u\left(\Phi^{-1}(p)\right)=\int_{Q} G(p, q) f\left(\Phi^{-1}(q)\right)\left|\left(\Phi^{-1}\right)^{\prime}(q)\right|^{2} d q .
$$

Since $f\left(\Phi^{-1}(\cdot)\right)\left|\left(\Phi^{-1}\right)^{\prime}(\cdot)\right|^{2}$ is bounded and locally Lipschitz continuous in $Q$ and since $f\left(\Phi^{-1}(q)\right)\left|\left(\Phi^{-1}\right)^{\prime}(q)\right|^{2}=\mathcal{O}\left(|q|^{-2-2 \beta(N-2)}\right)$ as $|q| \rightarrow \infty$, we claim from the regularity theorem for the Newtonian potential (see [4, Lemmas 4.1 and 4.2]) that $u\left(\Phi^{-1}(\cdot)\right) \in C^{1}(\bar{Q}) \cap C^{2}(Q)$. This implies that $u \in H_{\text {comp }}^{1}\left(\Pi_{\alpha}^{1}\right) \cap C^{2}\left(\Pi_{\alpha}^{1}\right) \cap L^{\infty}\left(\Pi_{\alpha}^{1}\right)$, $\left.u\right|_{\Lambda_{ \pm}} \in C^{1}\left(\overline{\Lambda_{ \pm}} \backslash\left\{e^{i \alpha}\right\}\right)$, and $\left|w-e^{i \alpha}\right|^{1 / 2} \nabla u(w) \in L^{\infty}\left(D\left(e^{i \alpha}, 1 / 2\right)\right)$.

Finally we prepare harmonic functions in $\Pi_{\alpha}^{1}$ which we need in the next section. For $j \in \mathbf{Z}_{+}$, we put

$$
V_{j}^{ \pm}(\eta)=\operatorname{Im}\left(\eta^{ \pm 1 \pm 2 j}\right)
$$

We immediately see that

$$
\begin{gathered}
\Delta V_{j}^{ \pm}=0 \quad \text { in } Q \\
V_{j}^{ \pm}(\cdot, 0)=0 \quad \text { on } \mathbf{R}_{+}, \quad \frac{\partial}{\partial \eta_{1}} V_{j}^{ \pm}(0, \cdot)=0 \quad \text { on } \mathbf{R}_{+} .
\end{gathered}
$$

We define

$$
Y_{j}^{ \pm}(\xi)=V_{j}^{ \pm}(\Phi(\xi))
$$

Since $\Phi$ is a conformal map from $\Pi_{\alpha}^{1}$ onto $Q$ which maps $\Upsilon_{1}$ and $\Upsilon_{2, \alpha}$ onto $\mathbf{R}_{+}$and $i \mathbf{R}_{+}$, respectively, we have

$$
\begin{gathered}
\Delta Y_{j}^{ \pm}=0 \quad \text { in } \Pi_{\alpha}^{1} \\
Y_{j}^{ \pm}=0 \quad \text { on } \Upsilon_{1}, \quad \frac{\partial}{\partial n_{+}} Y_{j}^{ \pm}(\xi)=\frac{\partial}{\partial n_{-}} Y_{j}^{ \pm}(\xi)=0 \quad \text { for } \xi \in \Upsilon_{2, \alpha} .
\end{gathered}
$$


We put

$$
\Lambda_{+}=\mathbf{K}_{\alpha}, \quad \Lambda_{-}=P \backslash \overline{\mathbf{K}_{\alpha}} .
$$

Using (2.9), (2.10), (2.15), and (2.16), we infer that the function $Y_{j}^{ \pm}(\xi)$ admits the following power series expansions for $\rho>1$ which can be differentiated term by term infinitely many times.

$$
\begin{aligned}
& Y_{j}^{+}(\xi)= \begin{cases}\sum_{k=0}^{\infty} A_{j, k}^{+} \rho^{\frac{2 j-2 k+1}{2 \beta}} \sin \frac{(2 j-2 k+1) \theta}{2 \beta} & \text { for } \xi \in \Lambda_{+}, \\
\sum_{k=0}^{\infty} B_{j, k}^{+} \rho^{\frac{-2 k-1}{2(1-\beta)}} \sin \frac{(-2 k-1)(\pi-\theta)}{2(1-\beta)} & \text { for } \xi \in \Lambda_{-},\end{cases} \\
& Y_{j}^{-}(\xi)= \begin{cases}\sum_{k=0}^{\infty} A_{j, k}^{-} \rho^{\frac{2 j-2 k+1}{2(1-\beta)}} \sin \frac{(2 j-2 k+1)(\pi-\theta)}{2(1-\beta)} & \text { for } \xi \in \Lambda_{-}, \\
\sum_{k=0}^{\infty} B_{j, k}^{-} \rho^{\frac{-2 k-1}{2 \beta}} \sin \frac{(-2 k-1) \theta}{2 \beta} & \text { for } \xi \in \Lambda_{+} .\end{cases}
\end{aligned}
$$

Let us evaluate the coefficients $A_{j, 0}^{+}, A_{0,1}^{+}, A_{j, 0}^{-}$, and $A_{0,1}^{-}$.

Proposition 2.3. We have

$$
\begin{gathered}
A_{j, 0}^{+}=(1-\beta)^{-\frac{1+2 j}{2 \beta}}, \quad A_{0,1}^{+}=\frac{1}{2}(1-\beta)^{-1+\frac{1}{2 \beta}}, \\
A_{j, 0}^{-}=-\beta^{-\frac{1+2 j}{2(1-\beta)}}, \quad A_{0,1}^{-}=-\frac{1}{2} \beta^{\frac{2 \beta-1}{2(1-\beta)}} .
\end{gathered}
$$

Proof. Plugging (2.2) and (2.8) into the equation $H\left(H^{-1}(z)\right)=z$, and identifying the powers of $(-z)$, we get

$$
p_{1}=\beta^{\frac{1}{1-\beta}}, \quad p_{2}=-\beta^{\frac{1+\beta}{1-\beta}} .
$$

Using (2.3), (2.8), (2.15), and (2.19), we obtain (2.18). The proof of (2.17) is similar.

It is convenient to normalize the functions $Y_{j}^{ \pm}(\xi)(j \geq 0)$. We define harmonic functions $X_{j}^{ \pm}(\xi)\left(j \in \mathbf{Z}_{+}\right)$by the recurrence formulae

$$
\begin{gathered}
X_{0}^{ \pm}(\xi)=\left(A_{0,0}^{ \pm}\right)^{-1} Y_{0}^{ \pm}(\xi) \\
X_{j}^{ \pm}(\xi)=\left(A_{j, 0}^{ \pm}\right)^{-1}\left(Y_{j}^{ \pm}(\xi)-\sum_{k=1}^{j} A_{j, k}^{ \pm} X_{j-k}^{ \pm}(\xi)\right) \quad \text { for } j \geq 1
\end{gathered}
$$


Then $X_{j}^{ \pm}(\xi)$ admits the following power series expansions for $\rho>1$.

$$
\begin{aligned}
& X_{j}^{+}(\xi)= \begin{cases}\rho^{\frac{2 j+1}{2 \beta}} \sin \frac{(2 j+1) \theta}{2 \beta}+\sum_{k=0}^{\infty} \tilde{A}_{j, k}^{+} \rho^{\frac{-1-2 k}{2 \beta}} \sin \frac{(-1-2 k) \theta}{2 \beta} & \text { on } \Lambda_{+}, \\
\sum_{k=0}^{\infty} \tilde{B}_{j, k}^{+} \rho^{\frac{-2 k-1}{2(1-\beta)}} \sin \frac{(-2 k-1)(\pi-\theta)}{2(1-\beta)} & \text { on } \Lambda_{-},\end{cases} \\
& X_{j}^{-}(\xi)= \begin{cases}\rho^{\frac{2 j+1}{2(1-\beta)}} \sin \frac{(2 j+1)(\pi-\theta)}{2(1-\beta)}+\sum_{k=0}^{\infty} \tilde{A}_{j, k}^{-} \rho^{\frac{-1-2 k}{2(1-\beta)}} \sin \frac{(-1-2 k)(\pi-\theta)}{2(1-\beta)} & \text { on } \Lambda_{-}, \\
\sum_{k=0}^{\infty} \tilde{B}_{j, k}^{-} \rho^{\frac{-2 k-1}{2 \beta}} \sin \frac{(-2 k-1) \theta}{2 \beta} & \text { on } \Lambda_{+},\end{cases}
\end{aligned}
$$

where

$$
\tilde{A}_{0,0}^{+}=\frac{1}{2}(1-\beta)^{-1+\frac{1}{\beta}}
$$

\section{Matching procedure}

In this section, our main aim is to prove the following theorem.

TheOREM 3.1. There exist sequences $\left\{\Psi_{j, k, l}^{+}\right\}_{j \geq 1, k \geq 0, l \geq 0},\left\{\Psi_{j, k, l}^{-}\right\}_{j \geq 1, k \geq 1, l \geq 0}$, $\left\{v_{j, k, l}\right\}_{j \geq 1, k \geq 0, l \geq 0}$, and $\left\{\lambda_{m, n, p}\right\}_{m \geq 1, n \geq 0, p \geq 0}$ satisfying $(0.15),(0.16),(0.17)$, and the conditions (i) and (ii) below.

(i) The functions $\Psi_{j, k, l}^{+}(x) \in J_{j}^{+}, \Psi_{j, k, l}^{-}(x) \in J_{k}^{-}$admit the following asymptotic expansions as $r \rightarrow 0$ which can be differentiated term by term arbitrarily many times.

$$
\begin{gathered}
\Psi_{l, m, n}^{+}(x) \sim \sum_{j=0}^{\infty} \sum_{k=0}^{\infty} C_{l, m, n, j, k}^{+} r^{\frac{2 j-2 l+1}{2 \beta}+2 k} \sin \frac{(2 j-2 l+1) \theta}{2 \beta} . \\
\Psi_{l, m, n}^{-}(x) \sim \sum_{j=0}^{\infty} \sum_{k=0}^{\infty} C_{l, m, n, j, k}^{-} r^{\frac{2 j-2 m+1}{2(1-\beta)}+2 k} \sin \frac{(2 j-2 m+1)(\pi-\theta)}{2(1-\beta)} .
\end{gathered}
$$

The function $v_{j, k, l}(\xi)$ admits the following asymptotic expansions as $\rho \rightarrow \infty$ which can be differentiated term by term once.

$$
\begin{aligned}
v_{l, m, n}(\xi) \sim \delta_{m, 0} C_{l, n}^{+} \rho^{\frac{2 l-1}{2 \beta}+2 n} \sin \frac{(2 l-1) \theta}{2 \beta} & \\
& +\sum_{k=0}^{\infty} \sum_{s=0}^{n} K_{l, m, n, k, s}^{+} \rho^{\frac{2 l-2 k-3}{2 \beta}+2 s} \sin \frac{(2 l-2 k-3) \theta}{2 \beta} \text { on } \Lambda_{+},
\end{aligned}
$$




$$
v_{l, m, n}(\xi) \sim \sum_{k=0}^{\infty} \sum_{s=0}^{n} K_{l, m, n, k, s}^{-} \rho^{\frac{2 m-2 k-1}{2(1-\beta)}+2 s} \sin \frac{(2 m-2 k-1)(\pi-\theta)}{2(1-\beta)} \text { on } \Lambda_{-} \text {, }
$$

where $\delta_{p, q}$ is Kronecker's symbol. Moreover we have $v_{j, k, l} \in H_{\text {comp }}^{1}\left(\Pi_{\alpha}^{1}\right) \cap C^{2}\left(\Pi_{\alpha}^{1}\right)$, $\left|\xi-e^{i \alpha}\right|^{1 / 2} \nabla v_{j, k, l}(\xi) \in L^{\infty}\left(D\left(e^{i \alpha}, 1 / 2\right)\right)$, and $\left.v_{j, k, l}\right|_{\Lambda_{ \pm}} \in C^{1}\left(\overline{\Lambda_{ \pm}} \backslash\left\{e^{i \alpha}\right\}\right)$.

(ii) For $l \geq 1, m \geq 0, n \geq 0, k \geq 0$, and $0 \leq s \leq n$, the following matching conditions hold.

$$
\begin{gathered}
K_{l, m, n, k, s}^{+}=C_{k+1, m, n-s, l-1, s}^{+} . \\
K_{l, m, n, k, s}^{-}=C_{l, k+1, n-s, m, s^{\circ}}^{-}
\end{gathered}
$$

Note. The condition $(3.5)_{l, m, n, k, s}$ is obtained as follows. Inserting $(3.1)_{l, m, n}$ $(l \geq 1, m \geq 0, n \geq 0)$ to (0.12), we obtain a formal power series of $\epsilon$ and $r$. Plugging $(3.3)_{l, m, n}(l \geq 1, m \geq 0, n \geq 0)$ to $(0.14)$ and using $\rho=\epsilon^{-1} r$, we also obtain a formal power series of $\epsilon$ and $r$. Identifying the coefficients of these two formal power series of $\epsilon$ and $r$, we get $(3.5)_{l, m, n, k, s}$. Similarly, we obtain $(3.6)_{l, m, n, k, s}$ by using (0.13), (0.14), $(3.2)_{l, m, n}$, and $(3.4)_{l, m, n}$.

We put

$$
v_{1,0,0}(\xi)=C_{1,0}^{+} X_{0}^{+}(\xi) .
$$

The above theorem immediately follows from the following lemma and induction.

Lemma 3.2. Let $L+1, K+1, J \in \mathbf{Z}_{+}$. Assume that there exist sequences

$$
\begin{array}{lll}
\left\{\Psi_{j, k, l}^{+}\right\}_{j \geq 1, k \geq 0,0 \leq l \leq L}, & \left\{\Psi_{j, k, L+1}^{+}\right\}_{0 \leq k \leq K, j \geq 1}, & \left\{\Psi_{j, K+1, L+1}^{+}\right\}_{1 \leq j \leq J-1}, \\
\left\{\Psi_{j, k, l}^{-}\right\}_{j \geq 1, k \geq 1,0 \leq l \leq L}, & \left\{\Psi_{j, k, L+1}^{-}\right\}_{1 \leq k \leq K+1, j \geq 1}, & \left\{\Psi_{j, K+2, L+1}^{-}\right\}_{1 \leq j \leq J-1}, \\
\left\{v_{j, k, l}\right\}_{j \geq 1, k \geq 0,0 \leq l \leq L}, & \left\{v_{j, k, L+1}\right\}_{0 \leq k \leq K, j \geq 1}, & \left\{v_{j, K+1, L+1}\right\}_{1 \leq j \leq J}, \\
\left\{\lambda_{j, k, l}\right\}_{j \geq 1, k \geq 0,0 \leq l \leq L}, & \left\{\lambda_{j, k, L+1}\right\}_{0 \leq k \leq K, j \geq 1}, & \left\{\lambda_{j, K+1, L+1}\right\}_{1 \leq j \leq J-1}
\end{array}
$$

which satisfy (0.15), (0.16), (0.17), (i) in Theorem 3.1, and the conditions

$$
\begin{array}{ll}
(3.5)_{l, m, n, k, s} & \text { for } l \geq 1, m \geq 0,0 \leq n \leq L, k \geq 0,0 \leq s \leq n, \\
(3.5)_{l, m, L+1, k, s} & \text { for } l \geq 1,0 \leq m \leq K, k \geq 0,0 \leq s \leq L+1, \\
(3.5)_{l, K+1, L+1, k, 0} & \text { for } 1 \leq l \leq J, 0 \leq k \leq J-2, \\
(3.5)_{l, K+1, L+1, k, s} & \text { for } 1 \leq l \leq J, 0 \leq k, 1 \leq s \leq L+1, \\
(3.6)_{l, m, n, k, s} & \text { for } l \geq 1, m \geq 0,0 \leq n \leq L, k \geq 0,0 \leq s \leq n, \\
(3.6)_{l, m, L+1, k, s} & \text { for } l \geq 1,0 \leq m \leq K, k \geq 0,1 \leq s \leq L+1, \\
(3.6)_{l, m, L+1, k, 0} & \text { for } l \geq 1,0 \leq m \leq K, 0 \leq k \leq K,
\end{array}
$$




$$
\begin{array}{ll}
(3.6)_{l, K+1, L+1, k, s} & \text { for } 1 \leq l \leq J, k \geq 0,1 \leq s \leq L+1, \\
(3.6)_{l, m, L+1, K+1,0} & \text { for } 1 \leq l \leq J-1,0 \leq m \leq K \\
(3.6)_{l, K+1, L+1, k, 0} & \text { for } 1 \leq l \leq J-1,0 \leq k \leq K+1, \\
(3.6)_{J, K+1, L+1, k, 0} & \text { for } 0 \leq k \leq K+1 .
\end{array}
$$

Then there exist $\Psi_{J, K+1, L+1}^{+}, \Psi_{J, K+2, L+1}^{-}, v_{J+1, K+1, L+1}$, and $\lambda_{J, K+1, L+1}$ satisfying (0.15), (0.16), (0.17), (i) in Theorem 3.1, and the conditions

$$
\begin{array}{ll}
(3.5)_{J+1, K+1, L+1, k, s} & \text { for } 0 \leq k, 1 \leq s \leq L+1, \\
(3.5)_{j+1, K+1, L+1, J-1,0} & \text { for } 0 \leq j \leq J-1 \\
(3.6)_{J+1, K+1, L+1, k, s} & \text { for } 0 \leq k, 1 \leq s \leq L+1, \\
(3.6)_{J, j, L+1, K+1,0} & \text { for } 0 \leq j \leq K+1
\end{array}
$$

Proof. We first construct $\Psi_{J, K+1, L+1}^{+}, \lambda_{J, K+1, L+1}$, and $\Psi_{J, K+2, L+1}^{-}$. It follows from Lemma 1.3 that there exist $\Psi_{J, K+1, L+1}^{+} \in J_{J}^{+}$and $\lambda_{J, K+1, L+1} \in \mathbf{R}$ satisfying (0.15) $J, K+1, L+1,(3.1)_{J, K+1, L+1}$, and $(3.5)_{j+1, K+1, L+1, J-1,0}$ for $0 \leq j \leq$ $J-1$. From Lemma 1.4, we claim that there exists $\Psi_{J, K+2, L+1}^{-} \in J_{K+2}^{-}$satisfying $(0.16)_{J, K+2, L+1},(3.2)_{J, K+2, L+1}$, and $(3.6)_{J, j, L+1, K+1,0}$ for $0 \leq j \leq K+1$.

Next we construct $v_{J+1, K+1, L+1}$, which solves the equation $(0.17)_{J+1, K+1, L+1}$. By $H(\xi)$ we denote the right side of $(0.17)_{J+1, K+1, L+1}$. The function $H(\xi)$ admits the following asymptotic expansions.

$$
\begin{gathered}
H(\xi) \sim-\lambda_{1}^{+} \delta_{K+1,0} C_{J+1, L}^{+} \rho^{\frac{2 J+1}{2 \beta}+2 L} \sin \frac{(2 J+1) \theta}{2 \beta} \\
+\sum_{k=0}^{\infty} \sum_{s=0}^{L} H_{k, s}^{+} \rho^{\frac{2 J-2 k-1}{2 \beta}+2 s} \sin \frac{(2 J-2 k-1) \theta}{2 \beta} \\
\text { as } \rho \rightarrow \infty, \quad \xi \in \Lambda_{+}, \\
H(\xi) \sim \sum_{k=0}^{\infty} \sum_{s=0}^{L} H_{k, s}^{-} \rho^{\frac{2 K-2 k-1}{2(1-\beta)}+2 s} \sin \frac{(2 K-2 k-1)(\pi-\theta)}{2(1-\beta)} \\
\text { as } \rho \rightarrow \infty, \quad \xi \in \Lambda_{-} .
\end{gathered}
$$

By $H^{+}(\xi)$ and $H^{-}(\xi)$ we denote the formal power series on the right side of (3.7) and that of (3.8), respectively. Let

$$
\begin{aligned}
L^{+}(\xi)= & \delta_{K+1,0} C_{J+1, L+1}^{+} \rho^{\frac{2 J+1}{2 \beta}+2(L+1)} \sin \frac{(2 J+1) \theta}{2 \beta} \\
& +\sum_{k=0}^{\infty} \sum_{s=1}^{L+1} L_{k, s}^{+} \rho^{\frac{2 J-2 k-1}{2 \beta}+2 s} \sin \frac{(2 J-2 k-1) \theta}{2 \beta}
\end{aligned}
$$


and

$$
L^{-}(\xi)=\sum_{k=0}^{\infty} \sum_{s=1}^{L+1} L_{k, s}^{-} \rho^{\frac{2 K-2 k+1}{2(1-\beta)}+2 s} \sin \frac{(2 K-2 k+1)(\pi-\theta)}{2(1-\beta)}
$$

be the formal power series which satisfy $\Delta L^{ \pm}(\xi)=H^{ \pm}(\xi)$. We set

$U=\left\{N \in \mathbf{N} ; T(N):=\min \left\{\frac{2 N-2 J+3}{2 \beta}-2 L, \frac{2 N-2 K+1}{2(1-\beta)}-2 L\right\} \geq 2+\frac{5}{\kappa}\right\}$.

For $N \in U$, we put

$$
\begin{aligned}
L_{N}^{+}(\xi)= & \delta_{K+1,0} C_{J+1, L+1}^{+} \rho^{\frac{2 J+1}{2 \beta}+2(L+1)} \sin \frac{(2 J+1) \theta}{2 \beta} \\
& +\sum_{k=0}^{N} \sum_{s=1}^{L+1} L_{k, s}^{+} \rho^{\frac{2 J-2 k-1}{2 \beta}+2 s} \sin \frac{(2 J-2 k-1) \theta}{2 \beta} \text { on } \Lambda^{+} \\
L_{N}^{-}(\xi)= & \sum_{k=0}^{N} \sum_{s=1}^{L+1} L_{k, s}^{-} \rho^{\frac{2 K-2 k+1}{2(1-\beta)}+2 s} \sin \frac{(2 K-2 k+1)(\pi-\theta)}{2(1-\beta)} \text { on } \Lambda^{-} \\
L_{N}^{ \pm}(\xi)= & 0 \text { on } \Lambda^{\mp} .
\end{aligned}
$$

We choose $\chi_{1} \in C^{\infty}[0, \infty)$ such that

$$
\chi_{1}=0 \text { on }[0,2], \quad \chi_{1}=1 \text { on }[3, \infty)
$$

We seek a solution $\tilde{v}_{N}$ to the equation $(0.17)_{J+1, K+1, L+1}$ which takes the form

$$
\tilde{v}_{N}=\chi_{1}(\rho) L_{N}^{+}(\xi)+\chi_{1}(\rho) L_{N}^{-}(\xi)+w_{N} .
$$

Inserting this into the equation $(0.17)_{J+1, K+1, L+1}$, we derive the equation for $w_{N}$ :

$$
\begin{gathered}
\Delta w_{N}=H_{N} \text { in } \Pi_{\alpha}^{1} \\
w_{N}=0 \text { on } \Upsilon_{1}, \quad \frac{\partial}{\partial n_{ \pm}} w_{N}(\xi)=0 \text { for } \xi \in \Upsilon_{2, \alpha}
\end{gathered}
$$

where

$$
\begin{aligned}
H_{N}(\xi)= & H(\xi)-\chi_{1}(\rho) \Delta_{\xi}\left(L_{N}^{+}(\xi)+L_{N}^{-}(\xi)\right) \\
& -2 \nabla_{\xi} \chi_{1}(\rho) \cdot \nabla_{\xi}\left(L_{N}^{+}(\xi)+L_{N}^{-}(\xi)\right)-2\left(L_{N}^{+}(\xi)+L_{N}^{-}(\xi)\right) \Delta_{\xi} \chi_{1}(\rho)
\end{aligned}
$$

We claim that $H_{N}(\xi)$ admits the asymptotic expansions as $\rho \rightarrow \infty$ :

$$
H_{N}(\xi) \sim \begin{cases}\sum_{k=N+1}^{\infty} \sum_{s=0}^{L} H_{k, s}^{+} \rho^{\frac{2 J-2 k-1}{2 \beta}+2 s} \sin \frac{(2 J-2 k-1) \theta}{2 \beta} & \text { on } \Lambda_{+}, \\ \sum_{k=N+1}^{\infty} \sum_{s=0}^{L} H_{k, s}^{-} \rho^{\frac{2 K-2 k+1}{2(1-\beta)}+2 s} \sin \frac{(2 K-2 k+1)(\pi-\theta)}{2(1-\beta)} & \text { on } \Lambda_{-} .\end{cases}
$$


Since $H_{N}(\xi)=\mathcal{O}\left(\rho^{-T(N)}\right)$ as $\rho \rightarrow \infty$, we infer from Proposition 2.2 that the equation (3.12) has a solution $w_{N}$ which admits the asymptotic expansions as $\rho \rightarrow$ $\infty$ :

$$
w_{N}(\xi)= \begin{cases}\sum_{j=1}^{M^{\prime}(N)} A_{j, k}^{+} \rho^{\frac{1-2 j}{2 \beta}} \sin \frac{(1-2 j) \theta}{2 \beta}+\mathcal{O}\left(\rho^{\frac{-1-2 M^{\prime}(N)}{2 \beta}}\right) & \text { on } \Lambda_{+}, \\ \sum_{j=1}^{M^{\prime}(N)} A_{j, k}^{-} \rho^{\frac{1-2 j}{2(1-\beta)}} \sin \frac{(1-2 j)(\pi-\theta)}{2(1-\beta)}+\mathcal{O}\left(\rho^{\frac{-1-2 M^{\prime}(N)}{2(1-\beta)}}\right) & \text { on } \Lambda_{-}\end{cases}
$$

where $M^{\prime}(N)=\left[\frac{\kappa}{2} T(N)-\kappa-\frac{1}{2}\right]-1$. We have $\lim _{N \rightarrow \infty} M^{\prime}(N)=\infty$.

Next we shall show that the function $\tilde{v}_{N}$ from (3.11) is independent of the choice of $N \in U$. We get

$$
\begin{gathered}
\Delta\left(\tilde{v}_{N}-\tilde{v}_{M}\right)=0 \text { in } \Pi_{\alpha}^{1}, \\
\tilde{v}_{N}-\tilde{v}_{M}=0 \quad \text { on } \Upsilon_{1}, \quad \frac{\partial}{\partial n_{ \pm}}\left(\tilde{v}_{N}-\tilde{v}_{M}\right)(\xi)=0 \text { for } \xi \in \Upsilon_{2, \alpha} .
\end{gathered}
$$

Since $\tilde{v}_{N}-\tilde{v}_{M}$ is bounded in $\Pi_{\alpha}^{1}$ for $N, M \in U$, we have $\tilde{v}_{N}-\tilde{v}_{M}=0$. Thus the function $\tilde{v}_{N}$ is independent of the choice of $N \in U$, which we denote by $\tilde{v}_{J+1, K+1, L+1}$. We define

$$
\begin{aligned}
v_{J+1, K+1, L+1}(\xi) & \\
= & \tilde{v}_{J+1, K+1, L+1}(\xi)+\delta_{K+1,0} \delta_{L+1,0} C_{J+1,0}^{+} X_{J}^{+}(\xi) \\
& +\sum_{k=0}^{J-1} C_{k+1, K+1, L+1, J, 0}^{+} X_{k}^{+}(\xi)+\sum_{k=0}^{K} C_{J+1, k+1, L+1, K+1,0}^{-} X_{k}^{-}(\xi)
\end{aligned}
$$

Then $v_{J+1, K+1, L+1}(\xi)$ admits the asymptotic expansions $(3.3)_{J+1, K+1, L+1}$ and $(3.4)_{J+1, K+1, L+1}$. Besides, $(3.5)_{J+1, K+1, L+1, k, 0}$ holds for $0 \leq k \leq J-1$ and (3.6) $)_{J+1, K+1, L+1, k, 0}$ holds for $0 \leq k \leq K$.

We shall prove that $(3.5)_{J+1, K+1, L+1, k, s}$ holds for $k \geq 0,1 \leq s \leq L+1$. Identifying the coefficients of $\rho^{\frac{2 J-2 k-1}{2 \beta}+2(s-1)} \sin \frac{(2 J-2 k-1) \theta}{2 \beta}$ in the asymptotic expansions of the both sides of $(0.17)_{J+1, K+1, L+1}$ as $\rho \rightarrow \infty, \xi \in \Lambda_{+}$, we get

$$
\begin{gathered}
\Delta\left(K_{J+1, K+1, L+1, k, s}^{+} \rho^{\frac{2 J-2 k-1}{2 \beta}+2 s} \sin \frac{(2 J-2 k-1) \theta}{2 \beta}\right) \\
=\left(-\lambda_{1}^{+} K_{J+1, K+1, L, k, s-1}^{+}-\lambda_{k+1, K+1, L+1-s} C_{J-k, s-1}^{+}\right. \\
\left.\quad-\sum_{m=1}^{J} \sum_{n=0}^{K+1} \sum_{p=0}^{L} \lambda_{m, n, p} K_{J+1-m, K+1-n, L-p, k-m, s-1}^{+}\right) \\
\times \rho^{\frac{2 J-2 k-1}{2 \beta}+2(s-1)} \sin \frac{(2 J-2 k-1) \theta}{2 \beta} .
\end{gathered}
$$


Equating the coefficients of $r^{\frac{2 J-2 k-1}{2 \beta}+2(s-1)} \sin \frac{(2 J-2 k-1) \theta}{2 \beta}$ in the asymptotic expansions of the both sides of $(0.15)_{k+1, K+1, L+1-s}$ as $r \rightarrow 0$, we get

$$
\begin{gathered}
\Delta\left(C_{k+1, K+1, L+1-s, J, s}^{+} r^{\frac{2 J-2 k-1}{2 \beta}+2 s} \sin \frac{(2 J-2 k-1) \theta}{2 \beta}\right) \\
=\left(-\sum_{m=1}^{k} \sum_{n=0}^{K+1} \sum_{p=0}^{L+1-s} \lambda_{m, n, p} C_{k+1-m, K+1-n, L+1-s-p, J-m, s-1}^{+}\right. \\
\left.-\lambda_{1}^{+} C_{k+1, K+1, L+1-s, J, s-1}^{+}-\lambda_{k+1, K+1, L+1-s} C_{J-k, s-1}^{+}\right) \\
\quad \times r^{\frac{2 J-2 k-1}{2 \beta}+2(s-1)} \sin \frac{(2 J-2 k-1) \theta}{2 \beta} .
\end{gathered}
$$

Since (3.5) $)_{l, m, n, k^{\prime}, s^{\prime}}$ holds for $l \geq 1, m \geq 0,0 \leq n \leq L, k^{\prime} \geq 0,0 \leq s^{\prime} \leq n$, it follows from (3.13) and (3.14) that

$$
\Delta_{x}\left(\left(K_{J+1, K+1, L+1, k, s}^{+}-C_{k+1, K+1, L+1-s, J, s}^{+}\right) r^{\frac{2 J-2 k-1}{2 \beta}+2 s} \sin \frac{(2 J-2 k-1) \theta}{2 \beta}\right)=0 .
$$

This implies that (3.5) $J+1, K+1, L+1, k, s$ holds for $k \geq 0,1 \leq s \leq L+1$. In a similar manner, we infer that $(3.6)_{J+1, K+1, L+1, k, s}$ holds for $k \geq 0,1 \leq s \leq L+1$. This completes the proof of Theorem 3.1.

Lemma 3.3. The number $\lambda_{1,0,0}$ is given by the formula $(0.8)$.

Proof. Using $(3.3)_{1,0,0},(3.5)_{1,0,0,0,0}$, and $v_{1,0,0}(\xi)=C_{1,0}^{+} X_{0}^{+}(\xi)$, we obtain

$$
C_{1,0,0,0,0}^{+}=K_{1,0,0,0,0}^{+}=C_{1,0}^{+} \tilde{A}_{0,0}^{+} .
$$

Since $\left(\Delta+\lambda_{1}^{+}\right) \Psi_{1,0,0}^{+}(x)=-\lambda_{1,0,0} \Psi_{0}^{+}(x)$ in $\Omega_{+}$, we have

$$
\begin{aligned}
\lambda_{1,0,0} & =-\lim _{\delta \rightarrow 0} \int_{\Omega_{+} \backslash D(0, \delta)} \Psi_{0}^{+}(x)\left(\Delta+\lambda_{1}^{+}\right) \Psi_{1,0,0}^{+}(x) d x \\
& =-\lim _{\delta \rightarrow 0} \int_{0}^{\alpha}\left(\Psi_{0}^{+}(\delta, \theta) \frac{\partial}{\partial r} \Psi_{1,0,0}^{+}(\delta, \theta)-\Psi_{1,0,0}^{+}(\delta, \theta) \frac{\partial}{\partial r} \Psi_{0}^{+}(\delta, \theta)\right) \delta d \theta \\
& =\frac{\pi}{2} C_{1,0,0,0,0}^{+} C_{1,0}^{+} \\
& =\frac{\pi}{2} \tilde{A}_{0,0}^{+}\left(C_{1,0}^{+}\right)^{2}
\end{aligned}
$$

where we used an integration by parts in the second line and we used (0.3) and $(3.1)_{1,0,0}$ in the third line. Combining this equality with $(2.20)$, we get the conclusion. 
Proof of Theorem 0.1. Let $N \in \mathbf{N}$. We define the approximate eigenvector of $L_{\epsilon}$ by the formula

$$
\begin{aligned}
\Phi_{\epsilon}^{N}(x)= & \chi\left(\epsilon^{-1 / 2} r\right) \sum_{j=1}^{N} \sum_{k=0}^{N} \sum_{l=0}^{N} \epsilon^{\frac{2 j-1}{2 \beta}+\frac{k}{1-\beta}+2 l} v_{j, k, l}(\xi) \\
& +\left(1-\chi\left(\epsilon^{-1 / 2} r\right)\right)\left(\Psi_{0}^{+}(x)+\sum_{j=1}^{N} \sum_{k=0}^{N} \sum_{l=0}^{N} \epsilon^{\frac{j}{\beta}+\frac{k}{1-\beta}+2 l} \Psi_{j, k, l}^{+}(x)\right) \\
& +\left(1-\chi\left(\epsilon^{-1 / 2} r\right)\right)\left(\sum_{j=1}^{N} \sum_{k=1}^{N} \sum_{l=0}^{N} \epsilon^{\frac{2 j-1}{2 \beta}+\frac{2 k-1}{2(1-\beta)}+2 l} \Psi_{j, k, l}^{-}(x)\right) .
\end{aligned}
$$

We immediately obtain $\Phi_{\epsilon}^{N}(x) \in \operatorname{Dom}\left(L_{\epsilon}\right)$ from the following claim.

ClaIM. Let $n_{ \pm}$be the interior unit normal to $\partial \Omega_{ \pm}$. Assume that $f \in Q_{\epsilon} \cap$ $C^{2}\left(\Omega_{\epsilon}\right),\left.f\right|_{\Omega_{ \pm}} \in C^{1}\left(\overline{\Omega_{ \pm}} \backslash\left\{\gamma(\epsilon), \gamma\left(t_{0}\right)\right\}\right), \Delta f \in L^{2}(\Omega), f=0$ on $\partial \Omega, \frac{\partial f}{\partial n_{ \pm}}(x)=0$ for $x \in \gamma\left(\left(\epsilon, t_{0}\right)\right)$, and $|x-\gamma(\epsilon)|^{1 / 2} \nabla f(x) \in L^{\infty}(D(\gamma(\epsilon), \epsilon / 2))$. Then we have $f \in \operatorname{Dom}\left(L_{\epsilon}\right)$.

We first prove this claim. Let $u \in Q_{\epsilon}$. A standard density argument shows that there exist two sequences $\left\{v_{j}^{+}\right\}_{j=1}^{\infty} \subset C^{\infty}\left(\overline{\Omega_{+}}\right)$and $\left\{v_{j}^{-}\right\}_{j=1}^{\infty} \subset C^{\infty}\left(\overline{\Omega_{-}}\right)$such that $\left.v_{j}^{ \pm} \rightarrow u\right|_{\Omega_{ \pm}}$in $H^{1}\left(\Omega_{ \pm}\right)$as $j \rightarrow \infty, v_{j}^{+}=v_{j}^{-}$on $\gamma((0, \epsilon))$, and $v_{j}^{ \pm}=0$ on a neighborhood of $\partial \Omega_{ \pm} \cap \partial \Omega$. We obtain

$$
\begin{aligned}
& (\nabla f, \nabla u)_{L^{2}(\Omega)} \\
& =\lim _{j \rightarrow \infty}\left\{\int_{\Omega_{+}} \nabla f \cdot \nabla v_{j}^{+} d x+\int_{\Omega_{-}} \nabla f \cdot \nabla v_{j}^{-} d x\right\} \\
& =\lim _{j \rightarrow \infty} \lim _{\delta \rightarrow+0}\left\{\int_{\Omega_{+} \backslash D(\gamma(\epsilon), \delta)} \nabla f \cdot \nabla v_{j}^{+} d x+\int_{\Omega_{-} \backslash D(\gamma(\epsilon), \delta)} \nabla f \cdot \nabla v_{j}^{-} d x\right\}
\end{aligned}
$$

by using $\left.f\right|_{\Omega_{ \pm}} \in C^{1}\left(\overline{\Omega_{ \pm}} \backslash\left\{\gamma(\epsilon), \gamma\left(t_{0}\right)\right\}\right) \cap C^{2}\left(\Omega_{ \pm}\right), \Delta f \in L^{2}(\Omega), v_{j}^{ \pm} \in C^{\infty}\left(\overline{\Omega_{ \pm}}\right)$, and Green's theorem

$$
\begin{aligned}
=\lim _{j \rightarrow \infty} \lim _{\delta \rightarrow+0}\{ & -\int_{\Omega_{+} \backslash D(\gamma(\epsilon), \delta)} u_{j}^{+} \Delta f d x-\int_{\Omega_{-} \backslash D(\gamma(\epsilon), \delta)} u_{j}^{-} \Delta f d x \\
& \left.-\int_{\Omega_{+} \cap \partial D(\gamma(\epsilon), \delta)} v_{j}^{+} \frac{\partial}{\partial N_{+}} f d S-\int_{\Omega_{-} \cap \partial D(\gamma(\epsilon), \delta)} v_{j}^{-} \frac{\partial}{\partial N_{-}} f d S\right\},
\end{aligned}
$$

where $N_{ \pm}$stands for the interior unit normal to $\partial\left(\Omega_{ \pm} \backslash D(\gamma(\epsilon), \delta)\right)$. Because the function $|x-\gamma(\epsilon)|^{1 / 2} \nabla f(x)$ is bounded in a neighborhood of $\gamma(\epsilon)$, we conclude that

$$
\lim _{\delta \rightarrow+0}\left\{\int_{\Omega_{+} \cap \partial D(\gamma(\epsilon), \delta)} v_{j}^{+} \frac{\partial}{\partial N_{+}} f d S+\int_{\Omega_{-} \cap \partial D(\gamma(\epsilon), \delta)} v_{j}^{-} \frac{\partial}{\partial N_{-}} f d S\right\}=0
$$


Hence we obtain

$$
(\nabla f, \nabla u)_{L^{2}(\Omega)}=-(u, \Delta f)_{L^{2}(\Omega)} \text { for all } u \in Q_{\epsilon} .
$$

Thus we get the assertion of the Claim.

Next we shall show that there exist $P>0$ and $Q \in \mathbf{R}$ such that the estimate

$$
\begin{gathered}
\left\|\left(\Delta_{x}+\lambda_{1}^{+}+\sum_{m=1}^{N} \sum_{n=0}^{N} \sum_{p=0}^{N} \lambda_{m, n, p} \epsilon^{\frac{m}{\beta}+\frac{n}{1-\beta}+2 p}\right) \Phi_{\epsilon}^{N}(x)\right\|_{L^{2}(\Omega)} \\
=\mathcal{O}\left(\epsilon^{P N+Q}\right) \text { as } \epsilon \rightarrow 0
\end{gathered}
$$

holds for all $N \in \mathbf{N}$. Using (0.15), (0.16), and (0.17), we obtain

$$
\left(\Delta_{x}+\lambda_{1}^{+}+\sum_{m=1}^{N} \sum_{n=0}^{N} \sum_{p=0}^{N} \epsilon^{\frac{m}{\beta}+\frac{n}{1-\beta}+2 p}\right) \Phi_{\epsilon}^{N}(x)=I_{\epsilon, 1}^{N}+I_{\epsilon, 2}^{N}+I_{\epsilon, 3}^{N}+I_{\epsilon, 4}^{N},
$$

where

$$
\begin{aligned}
I_{\epsilon, 1}^{N}= & \chi\left(\epsilon^{-1 / 2} r\right)\left\{\lambda_{1}^{+} \sum_{j=1}^{N} \sum_{k=0}^{N} \epsilon^{\frac{2 j-1}{2 \beta}+\frac{k}{1-\beta}+2 N} v_{j, k, N}(\xi)\right. \\
+ & \left.\sum_{m=1}^{N} \sum_{n=0}^{N} \sum_{p=0}^{N} \sum_{j=N-m+1}^{N} \sum_{k=N-n+1}^{N} \sum_{l=N-p}^{N} \epsilon^{\frac{2 m+2 j-1}{2 \beta}+\frac{k+n}{1-\beta}+2(p+l)} \lambda_{m, n, p} v_{j, k, l}(\xi)\right\} \\
I_{\epsilon, 2}^{N}= & \left(1-\chi\left(\epsilon^{-1 / 2} r\right)\right) \sum_{m=1}^{N} \sum_{n=0}^{N} \sum_{p=0}^{N} \sum_{j=N-m+1}^{N} \sum_{k=N-n+1}^{N} \sum_{l=N-p+1}^{N} \lambda_{m, n, p} \\
& \times\left(\epsilon^{\frac{m+j}{\beta}+\frac{n+k}{1-\beta}+2(p+l)} \Psi_{j, k, l}^{+}(x)+\epsilon^{\frac{2 m+2 j-1}{2 \beta}+\frac{2 n+2 k-1}{2(1-\beta)}+2(p+l)} \Psi_{j, k, l}^{-}(x)\right) \\
I_{\epsilon, 3}^{N}= & 2 \epsilon^{-1 / 2}(\nabla \chi)\left(\epsilon^{-1 / 2} r\right) \cdot \nabla_{x}\left\{\sum_{j=1}^{N} \sum_{k=0}^{N} \sum_{l=0}^{N} \epsilon^{\frac{2 j-1}{2 \beta}+\frac{k}{1-\beta}+2 l} v_{j, k, l}(\xi)-\Psi_{0}^{+}(x)\right. \\
& \left.-\sum_{j=1}^{N} \sum_{k=0}^{N} \sum_{l=0}^{N} \epsilon^{\frac{j}{\beta}+\frac{k}{1-\beta}+2 l} \Psi_{j, k, l}^{+}(x)-\sum_{j=1}^{N} \sum_{k=1}^{N} \sum_{l=0}^{N} \epsilon^{\frac{2 j-1}{2 \beta}+\frac{2 k-1}{2(1-\beta)}+2 l} \Psi_{j, k, l}^{-}(x)\right\} \\
& \left.-\sum_{j=1}^{N} \sum_{k=0}^{N} \sum_{l=0}^{N} \epsilon^{\frac{j}{\beta}+\frac{k}{1-\beta}+2 l} \Psi_{j, k, l}^{+}(x)-\sum_{j=1}^{N} \sum_{k=1}^{N} \sum_{l=0}^{N} \epsilon^{\frac{2 j-1}{2 \beta}+\frac{2 k-1}{2(1-\beta)}+2 l} \Psi_{j, k, l}^{-}(x)\right\} \\
I_{\epsilon, 4}^{N}= & \epsilon^{-1}(\Delta \chi)\left(\epsilon^{-1 / 2} r\right)\left\{\sum_{j=1}^{N} \sum_{k=0}^{N} \sum_{l=0}^{N} \epsilon^{\frac{2 j-1}{2 \beta}+\frac{k}{1-\beta}+2 l} v_{j, k, l}(\xi)-\Psi_{0}^{+}(x)\right. \\
& \\
& \\
&
\end{aligned}
$$


So it suffices to show that, for $j=1,2,3,4$, there exist $P_{j}>0$ and $Q_{j} \in \mathbf{R}$ such that the estimate

$$
\left\|I_{\epsilon, j}^{N}\right\|_{L^{2}(\Omega)}=\mathcal{O}\left(\epsilon^{P_{j} N+Q_{j}}\right) \quad \text { as } \epsilon \rightarrow 0
$$

holds for all $N \in \mathbf{N}$.

We first estimate $I_{\epsilon, 1}^{N}$. By (i) of Theorem 3.1 we have

$$
\left|v_{j, k, l}(\xi)\right| \leq C\left(1+\rho^{\frac{2 j-1}{2 \beta}}+\rho^{\frac{2 k-1}{2(1-\beta)}}\right) \text { in } \Pi_{\alpha}^{1}
$$

Thus we obtain

$$
\begin{aligned}
& \left\|\chi\left(\epsilon^{-1 / 2} r\right) v_{j, k, l}(\xi)\right\|_{L^{2}(\Omega)} \\
& \quad \leq C\left\{\int_{|x| \leq 2 \epsilon^{1 / 2}}\left(1+\left|\epsilon^{-1} x\right|^{\frac{2 j-1}{\beta}}+\left|\epsilon^{-1} x\right|^{\frac{2 k-1}{1-\beta}}\right)^{2} d x\right\}^{1 / 2} \\
& \quad \leq C\left(\epsilon^{1 / 8}+\epsilon^{-\frac{2 j-1}{2 \beta}+\frac{1}{2}}+\epsilon^{-\frac{2 k-1}{2(1-\beta)}+\frac{1}{2}}\right) .
\end{aligned}
$$

Using this estimate, we get $\left\|I_{\epsilon, 1}^{N}\right\|_{L^{2}(\Omega)} \leq C \epsilon^{2 N}$.

Next we estimate $I_{\epsilon, 2}^{N}$. By (i) of Theorem 3.1 we have

$$
\begin{aligned}
& \left|\Psi_{j, k, l}^{+}(x)\right| \leq C r^{\frac{-2 j+1}{2 \beta}} \quad \text { on } \Omega_{+} \cap D\left(0, r_{0}\right), \\
& \left|\Psi_{j, k, l}^{-}(x)\right| \leq C r^{\frac{-2 k+1}{2(1-\beta)}} \quad \text { on } \quad \Omega_{-} \cap D\left(0, r_{0}\right) .
\end{aligned}
$$

Thus we get

$$
\begin{aligned}
& \left\|\left(1-\chi\left(\epsilon^{-1 / 2} r\right)\right) \Psi_{j, k, l}^{+}(x)\right\|_{L^{2}\left(\Omega_{+}\right)} \leq C\left(1+\epsilon^{\frac{-2 j+1}{4 \beta}+\frac{1}{2}}\right) \\
& \left\|\left(1-\chi\left(\epsilon^{-1 / 2} r\right)\right) \Psi_{j, k, l}^{-}(x)\right\|_{L^{2}\left(\Omega_{-}\right)} \leq C\left(1+\epsilon^{\frac{-2 k+1}{4(1-\beta)}+\frac{1}{2}}\right) .
\end{aligned}
$$

Thanks to these estimates, we obtain $\left\|I_{\epsilon, 2}^{N}\right\|_{L^{2}(\Omega)} \leq C \epsilon^{2 N}$.

Next we estimate $I_{\epsilon, 4}^{N}$. It follows from (ii) of Theorem 3.1 that

$$
\begin{aligned}
I_{\epsilon, 4}^{N}=\epsilon^{-1}(\Delta \chi)\left(\epsilon^{-1 / 2} r\right)\{ & \sum_{j=1}^{N} \sum_{k=0}^{N} \sum_{l=0}^{N} \epsilon^{\frac{2 j-1}{2 \beta}+\frac{k}{1-\beta}+2 l} \tilde{v}_{j, k, l}^{+}(\xi) \\
& \left.-\tilde{\Psi}_{0}(x)-\sum_{j=1}^{N} \sum_{k=0}^{N} \sum_{l=0}^{N} \epsilon^{\frac{j}{\beta}+\frac{k}{1-\beta}+2 l} \tilde{\Psi}_{j, k, l}^{+}(x)\right\} \text { on } \Omega_{+},
\end{aligned}
$$

where

$$
\begin{gathered}
\tilde{v}_{j, k, l}^{+}(\xi)=v_{j, k, l}(\xi)-\sum_{p=0}^{N-1} \sum_{s=0}^{l} K_{j, k, l, p, s}^{+} \rho^{\frac{2 j-2 p-3}{2 \beta}+2 s} \sin \frac{(2 j-2 p-3) \theta}{2 \beta} \\
-\delta_{k, 0} C_{j, l} \rho^{\frac{2 j-1}{2 \beta}+2 l} \sin \frac{(2 j-1) \theta}{2 \beta}
\end{gathered}
$$




$$
\begin{gathered}
\tilde{\Psi}_{0}(x)=\Psi_{0}^{+}(x)-\sum_{j=1}^{N} \sum_{l=0}^{N} C_{j, l}^{+} r^{\frac{2 j-1}{2 \beta}+2 l} \sin \frac{(2 j-1) \theta}{2 \beta} \\
\tilde{\Psi}_{j, k, l}^{+}(x)=\Psi_{j, k, l}^{+}(x)-\sum_{p=0}^{N-1} \sum_{s=0}^{N-l} C_{j, k, l, p, s}^{+} r^{\frac{2 p-2 j+1}{2 \beta}+2 s} \sin \frac{(2 p-2 j+1) \theta}{2 \beta} .
\end{gathered}
$$

By (i) of Theorem 3.1 we have

$$
\begin{aligned}
\left|\tilde{v}_{j, k, l}(\xi)\right| & \leq C \rho^{\frac{2 j-2 N-3}{2 \beta}+2 l} \text { for } \xi \in \Lambda_{+}, \quad \rho \geq 1, \\
\left|\tilde{\Psi}_{0}(x)\right| & \leq C r^{(2 N+1) \min \left\{1, \frac{1}{2 \beta}\right\}-\frac{1}{\beta}} \text { on } \Omega_{+} \cap D\left(0, r_{0}\right), \\
\left|\tilde{\Psi}_{j, k, l}^{+}(x)\right| & \leq C r^{\frac{-2 j+1}{2 \beta}+(N-l+1) \min \left\{\frac{1}{\beta}, 2\right\}} \text { on } \Omega_{+} \cap D\left(0, r_{0}\right) .
\end{aligned}
$$

Using these estimates, we have

$$
\left\|I_{\epsilon, 4}^{N}\right\|_{L^{2}\left(\Omega_{+}\right)} \leq C \epsilon^{\frac{N}{2} \min \left\{\frac{1}{\beta}, 2\right\}-1} .
$$

Similarly, we get

$$
\left\|I_{\epsilon, 4}^{N}\right\|_{L^{2}\left(\Omega_{-}\right)} \leq C \epsilon^{\frac{N}{2} \min \left\{\frac{1}{1-\beta}, 2\right\}-1} .
$$

Therefore $(3.16)_{4}$ holds.

The proof of $(3.16)_{3}$ is similar to that of $(3.16)_{4}$. Thus we conclude that the estimate (3.15) holds.

It is readily seen that

$$
\left\|\Phi_{\epsilon}^{N}\right\|_{L^{2}(\Omega)}=1+o(1) \quad \text { as } \epsilon \rightarrow 0 .
$$

Combining this with (3.15) and the fact that $\Phi_{\epsilon}^{N} \in \operatorname{Dom}\left(L_{\epsilon}\right)$, we get

$$
\begin{aligned}
& \operatorname{dist}\left(\sigma\left(L_{\epsilon}\right), \lambda_{1}^{+}+\sum_{m=1}^{N} \sum_{n=0}^{N} \sum_{p=0}^{N} \lambda_{m, n, p} \epsilon^{\frac{m}{\beta}+\frac{n}{1-\beta}+2 p}\right) \\
& \leq\left\|\left(\Delta_{x}+\lambda_{1}^{+}+\sum_{m=1}^{N} \sum_{n=0}^{N} \sum_{p=0}^{N} \lambda_{m, n, p} \epsilon^{\frac{m}{\beta}+\frac{n}{1-\beta}+2 p}\right) \Phi_{\epsilon}^{N}(x)\right\|_{L^{2}(\Omega)} /\left\|\Phi_{\epsilon}^{N}(x)\right\|_{L^{2}(\Omega)} \\
& =\mathcal{O}\left(\epsilon^{P N+Q}\right)
\end{aligned}
$$

On the other hand, we have $\lambda_{2}(\epsilon) \geq \min \left\{\lambda_{2}^{+}, \lambda_{1}^{-}\right\}>\lambda_{1}^{+}$for $\epsilon \in\left(0, t_{0}\right]$, because $Q_{\epsilon} \subset Q^{+} \oplus Q^{-}$. This together with the estimate (3.17) implies that

$$
\lambda_{1}^{+}(\epsilon)=\lambda_{1}^{+}+\sum_{m=1}^{N} \sum_{n=0}^{N} \sum_{p=0}^{N} \lambda_{m, n, p} \epsilon^{\frac{m}{\beta}+\frac{n}{1-\beta}+2 p}+\mathcal{O}\left(\epsilon^{P N+Q}\right) \quad \text { as } \epsilon \rightarrow 0 .
$$

The proof is complete. 


\section{Proof of Theorem 0.2}

In this section we demonstrate Theorem 0.2 .

Proof of Theorem 0.2. The proof of this theorem is a slight modification of that of Theorem 0.1. We assume that $\beta \notin \Gamma, \lambda_{1}^{+}=\lambda_{1}^{-}$, and $\alpha>\pi / 2$. We seek the approximate second eigenvalue of $L_{\epsilon}$ and the associated approximate eigenvector in the forms $(0.11)-(0.14)$. As in the proofs of Theorem 1.3 and Lemma 3.3, we obtain the necessary and sufficient condition for the equation $(0.16)_{j, k, l}$ to admit a solution $\Psi_{j, k, l}^{-} \in J_{k}^{-}$satisfying $(3.2)_{j, k, l}$ and $(3.6)_{j, p, l, k-1,0}$ for $0 \leq p \leq k-1$ :

$$
\begin{aligned}
\sum_{m=1}^{j-1} \sum_{n=0}^{k-1} & \sum_{p=0}^{l} \lambda_{m, n, p}\left(\Psi_{j-m, k-n, l-p}^{-}, \Psi_{0}^{-}\right)_{\Omega_{-}} \\
& -\sum_{p=0}^{k-1} \frac{(2 p-2 k+1) \pi}{2} K_{j, p, l, k-1,0}^{-} C_{k-p, 0}^{-}=0 .
\end{aligned}
$$

By induction, we shall construct the coefficients of $(0.11)-(0.14)$ which satisfy the statements in Theorem 3.1 together with the solvability condition $(4.1)_{j, k, l}$ for $j \geq 1$, $k \geq 1$, and $l \geq 0$. We put $v_{1,0,0}(\xi)=C_{1,0}^{+} X_{0}^{+}(\xi)$. Suppose that the assumptions in Lemma 3.2 and (4.1) $)_{J, K+2, L+1}$ hold. As in the proof of Lemma 3.2, we obtain $\Psi_{J, K+1, L+1}^{+}, \tilde{\Psi}_{J, K+2, L+1}^{-}, \tilde{v}_{J+1, K+1, L+1}$, and $\lambda_{J, K+1, L+1}$ satisfying the following conditions (a) and (b), which are the same as the conclusion of Lemma 3.2 except for the substitution for the notations.

(a) The functions $\Psi_{J, K+1, L+1}^{+}, \tilde{\Psi}_{J, K+2, L+1}^{-}$, and $\tilde{v}_{J+1, K+1, L+1}$ solve the equations $(0.16)_{J, K+1, L+1},(0.17)_{J, K+2, L+1}$, and $(0.18)_{J+1, K+1, L+1}$, respectively. The function $\Psi_{J, K+1, L+1}^{+}(x) \in J_{J}^{+}$admits the asymptotic expansion (3.1) $)_{J, K+1, L+1}$. The function $\tilde{\Psi}_{J, K+2, L+1}^{-}(x) \in J_{K+2}^{-}$admits the following asymptotic expansion as $r \rightarrow 0$ :

$$
\tilde{\Psi}_{J, K+2, L+1}^{-}(x) \sim \sum_{j=0}^{\infty} \sum_{k=0}^{\infty} \tilde{C}_{J, K+2, L+1, j, k}^{-} r^{\frac{2 j-2 K-3}{2(1-\beta)}+2 k} \sin \frac{(2 j-2 K-3)(\pi-\theta)}{2(1-\beta)} .
$$

The function $\tilde{v}_{J+1, K+1, L+1}(\xi)$ admits the following asymptotic expansions as $\rho \rightarrow$ $\infty$ :

$$
\begin{aligned}
& \tilde{v}_{J+1, K+1, L+1}(\xi) \\
& \sim \delta_{K+1,0} C_{J+1, L+1}^{+} \rho^{\frac{2 J+1}{2 \beta}+2 L+2} \sin \frac{(2 J+1) \theta}{2 \beta} \\
& \quad+\sum_{k=0}^{\infty} \sum_{s=0}^{L+1} \tilde{K}_{J+1, K+1, L+1, k, s}^{+} \rho^{\frac{2 J-2 k-1}{2 \beta}+2 s} \sin \frac{(2 J-2 k-1) \theta}{2 \beta} \text { on } \Lambda_{+}
\end{aligned}
$$




$$
\begin{aligned}
& \tilde{v}_{J+1, K+1, L+1}(\xi) \\
& \sim \sum_{k=0}^{\infty} \sum_{s=0}^{L+1} \tilde{K}_{J+1, K+1, L+1, k, s}^{-} \rho^{\frac{2 K-2 k+1}{2(1-\beta)}+2 s} \sin \frac{(2 K-2 k+1)(\pi-\theta)}{2(1-\beta)} \text { on } \Lambda_{-} .
\end{aligned}
$$

Moreover, we have $\tilde{v}_{J+1, K+1, L+1} \in H_{\text {comp }}^{1}\left(\Pi_{\alpha}^{1}\right) \cap C^{2}\left(\Pi_{\alpha}^{1}\right)$ and $\left.\tilde{v}_{J+1, K+1, L+1}\right|_{\Lambda_{ \pm}} \in$ $C^{1}\left(\overline{\Lambda_{ \pm}} \backslash\left\{e^{i \alpha}\right\}\right)$.

(b) The following conditions hold.

$$
\begin{array}{ll}
\tilde{K}_{J+1, K+1, L+1, k, s}^{+}=C_{k+1, K+1, L+1-s, J, s}^{+} & \text {for } 0 \leq k, 1 \leq s \leq L+1 \\
(3.5)_{j+1, K+1, L+1, J-1,0} & \text { for } 0 \leq j \leq J \\
\tilde{K}_{J+1, K+1, L+1, k, s}^{-}=C_{J+1, k+1, L+1-s, K+1, s}^{-} & \text {for } 0 \leq k, 1 \leq s \leq L+1 \\
K_{J, j, L+1, K+1,0}^{-}=\tilde{C}_{J, K+2, L+1, j, 0}^{-} & \text {for } 0 \leq j \leq K+1
\end{array}
$$

For $\tau \in \mathbf{R}$, we put

$$
\begin{gathered}
\Psi_{J, K+2, L+1}^{-}(x)=\tilde{\Psi}_{J, K+2, L+1}^{-}(x)+\tau \Psi_{0}^{-}(x), \\
v_{J+1, K+1, L+1}(\xi)=\tilde{v}_{J+1, K+1, L+1}(\xi)+\tau C_{1,0}^{-} X_{0}^{-}(\xi) .
\end{gathered}
$$

Then we have the asymptotic expansions $(3.2)_{J, K+2, L+1},(3.3)_{J+1, K+1, L+1}$, and $(3.4)_{J+1, K+1, L+1}$. Moreover, the conclusions of Lemma 3.2 hold. Since

$$
K_{J+1, K+1, L+1, K+1,0}^{-}=\tilde{K}_{J+1, K+1, L+1, K+1,0}^{-}+C_{1,0}^{-} \tau \tilde{A}_{0,0}^{-},
$$

we infer that the condition $(4.1)_{J+1, K+2, L+1}$ is equivalent to the equation

$$
\begin{aligned}
\left(\lambda_{1,0,0}+\right. & \left.\tilde{A}_{0,0}^{-}\left(C_{1,0}^{-}\right)^{2}\right) \tau \\
=- & -\frac{\pi}{2} C_{1,0}^{-} \tilde{K}_{J+1, K+1, L+1, K+1,0}^{-} \\
& +\sum_{p=0}^{K} \frac{(2 p-2 K-3) \pi}{2} K_{J+1, p, L+1, K+1,0}^{-} C_{K+2-p, 0}^{-} \\
& -\sum_{\substack{1 \leq m \leq J \\
0 \leq n \leq K+1 \\
0 \leq p \leq L+1 \\
(m, n, p) \neq(1,0,0)}} \lambda_{m, n, p}\left(\Psi_{J+1-m, K+2-n, L+1-p}^{-}, \Psi_{0}^{-}\right)_{\Omega_{-}} .
\end{aligned}
$$

Because $\lambda_{1,0,0}+\tilde{A}_{0,0}^{-}\left(C_{1,0}^{-}\right)^{2}>0$ and the right side of (4.2) is independent of $\tau$, we claim that the equation (4.2) has a unique root $\tau \in \mathbf{R}$. Thus we obtain $\Psi_{J, K+1, L+1}^{+}$, $\Psi_{J, K+2, L+1}^{-}, v_{J+1, K+1, L+1}$, and $\lambda_{J, K+1, L+1}$ satisfying $(4.1)_{J+1, K+2, L+1}$ as well as the conclusions of Lemma 3.2.

The approximate first eigenvalue of $L_{\epsilon}$ and the associated approximate eigenvector are constructed in a similar manner. 


\section{Proofs of Theorems 0.3 and 0.4}

Because the proofs of Theorems 0.3 and 0.4 are similar to those of Theorems 0.1 and 0.2 , we demonstrate only Theorem 0.3 omitting details. Suppose that (A.1), (A.2), and $\beta \in \Gamma$ hold. Let $(q, s)$ be a unique pair of natural numbers such that

$$
\beta=\frac{q}{s}, \quad \operatorname{gcd}(q, s)=1 .
$$

We have

$$
q \equiv 1 \quad(\bmod 2), \quad s \equiv 0 \quad(\bmod 2)
$$

We put $t=s-q$. We obtain

$$
1-\beta=\frac{t}{s}, \quad t \in \mathbf{N}, \quad t \equiv 1 \cdot(\bmod 2), \quad \operatorname{gcd}(t, s)=1 .
$$

Let $\left(s^{\prime}, q^{\prime}, t^{\prime}\right) \in \mathbf{N}^{3}$ be such that

$$
s=2 s^{\prime}, \quad q=2 q^{\prime}-1, \quad t=2 t^{\prime}-1 .
$$

We seek the approximate first eigenvalue of $L_{\epsilon}$ and the associated approximate eigenvector in the following form.

$$
\begin{aligned}
\lambda(\epsilon) & =\lambda_{1}^{+}+\sum_{\substack{m \geq 1 \\
n, p, h \geq 0}} \lambda_{m, n, p, h} \epsilon^{\frac{m}{\beta}+\frac{n}{1-\beta}+2 p}\left(\epsilon^{s} \log \epsilon\right)^{h} \\
\Psi_{\epsilon}^{\text {out },+}(x) & =\Psi_{0}^{+}(x)+\sum_{\substack{j \geq 1 \\
k, l, g \geq 0}} \epsilon^{\frac{j}{\beta}+\frac{k}{1-\beta}+2 l}\left(\epsilon^{s} \log \epsilon\right)^{g} \Psi_{j, k, l, g}^{+}(x) \text { in } \Omega_{+} \backslash D(0, \sqrt{\epsilon}) \\
\Psi_{\epsilon}^{\text {out },-}(x) & =\sum_{\substack{j, k \geq 1 \\
l, g \geq 0}} \epsilon^{\frac{2 j-1}{2 \beta}+\frac{2 k-1}{2(1-\beta)}+2 l}\left(\epsilon^{s} \log \epsilon\right)^{g} \Psi_{j, k, l, g}^{-}(x) \text { in } \Omega_{-} \backslash D(0, \sqrt{\epsilon}) \\
\Psi_{\epsilon}^{\text {in }}(x) & =\sum_{\substack{j \geq 1 \\
k, l, g \geq 0}} \epsilon^{\frac{2 j-1}{2 \beta}+\frac{k}{1-\beta}+2 l}\left(\epsilon^{s} \log \epsilon\right)^{g} v_{j, k, l, g}(\xi) \text { in } \Omega_{\epsilon} \cap D(0,2 \sqrt{\epsilon})
\end{aligned}
$$

As in the derivation of the equations $(0.15)_{j, k, l}-(0.17)_{j, k, l}$, we obtain the following equations.

$$
\begin{aligned}
& \left(\Delta_{x}+\lambda_{1}^{+}\right) \Psi_{j, k, l, g}^{+}(x) \\
& =-\lambda_{j, k, l, g} \Psi_{0}^{+}(x)-\sum_{\substack{1 \leq m \leq j-1 \\
0 \leq n \leq k \\
0 \leq p \leq l \\
0 \leq h \leq g}} \lambda_{m, n, p, h} \Psi_{j-m, k-n, l-p, g-h}^{+}(x) \text { in } \Omega_{+}, \\
& \Psi_{j, k, l, g}^{+}(x)=0 \quad \text { on } \partial \Omega_{+} \cap \partial \Omega, \quad \frac{\partial}{\partial n} \Psi_{j, k, l, g}^{+}(x)=0 \quad \text { on } \gamma\left(\left(0, t_{0}\right)\right) .
\end{aligned}
$$




$$
\begin{aligned}
& \left(\Delta_{x}+\lambda_{1}^{+}\right) \Psi_{j, k, l, g}^{-}(x) \\
& =-\sum_{\substack{1 \leq m \leq j-1 \\
0 \leq n \leq k-1 \\
0 \leq p \leq l \\
0 \leq h \leq g}} \lambda_{m, n, p, h} \Psi_{j-m, k-n, l-p, g-h}^{-}(x) \text { in } \Omega_{-}, \\
& \Psi_{j, k, l, g}^{-}(x)=0 \quad \text { on } \partial \Omega_{-} \cap \partial \Omega, \quad \frac{\partial}{\partial n} \Psi_{j, k, l, g}^{-}(x)=0 \quad \text { on } \gamma\left(\left(0, t_{0}\right)\right) \text {. } \\
& \Delta_{\xi} v_{j, k, l, g}(\xi)=-\lambda_{1}^{+} v_{j, k, l-1, g}(\xi) \\
& -\sum_{\substack{1 \leq m \leq j-1 \\
0 \leq n \leq k \\
0 \leq p \leq l-1 \\
0 \leq h \leq g}} \lambda_{m, n, p, h} v_{j-m, k-n, l-p-1, g-h}(\xi) \text { in } \Pi_{\alpha}^{1}, \\
& v_{j, k, l, g}=0 \quad \text { on } \quad \Upsilon_{1}, \quad \frac{\partial}{\partial n_{ \pm}} v_{j, k, l, g}(\xi)=0 \quad \text { for } \xi \in \Upsilon_{2, \alpha}
\end{aligned}
$$

The assertion of Theorem 0.3 follows from the result below.

Theorem 5.1. There exist $\left\{\Psi_{j+1, k, l, g}^{+}\right\}_{j, k, l, g \geq 0}, \quad\left\{\Psi_{j+1, k+1, l, g}^{-}\right\}_{j, k, l, g \geq 0}$, $\left\{v_{j+1, k, l, g}\right\}_{j, k, l, g \geq 0}$, and $\left\{\lambda_{m+1, n, p, h}\right\}_{m, n, p, h \geq 0}$ satisfying $(5.5),(5.6),(5.7)$, and the conditions (i), (ii), and (iii) below.

(i) We have

$$
\begin{aligned}
& \Psi_{l, m, n, p}^{ \pm} \in C^{\infty}\left(\overline{\Omega_{ \pm}} \backslash\left\{0, \gamma\left(t_{0}\right)\right\}\right), \quad(1-\chi(r)) \Psi_{l, m, n, p}^{ \pm} \in L^{2}\left(\Omega_{ \pm}\right), \\
& \Psi_{l, m, n, p}^{ \pm}=0 \text { on } \partial \Omega_{ \pm} \cap \partial \Omega, \quad \frac{\partial}{\partial n} \Psi_{l, m, n, p}^{ \pm}=0 \text { on } \gamma\left(\left(0, t_{0}\right)\right) .
\end{aligned}
$$

We obtain

$$
v_{l, m, n, p} \in H_{\mathrm{comp}}^{1}\left(\Pi_{\alpha}^{1}\right) \cap C^{2}\left(\Pi_{\alpha}^{1}\right),\left.\quad v_{l, m, n, p}\right|_{\Lambda_{ \pm}} \in C^{1}\left(\overline{\Lambda_{ \pm}} \backslash\left\{e^{i \alpha}\right\}\right)
$$

and

$$
\left|\xi-e^{i \alpha}\right|^{1 / 2} \nabla v_{l, m, n, p}(\xi) \in L^{\infty}\left(D\left(e^{i \alpha}, 1 / 2\right)\right)
$$

(ii) The functions $\Psi_{l, m, n, p}^{+}(x)$ and $\Psi_{l, m, n, p}^{-}(x)$ admit the following asymptotic expansions as $r \rightarrow 0$ which can be differentiated term by term arbitrarily many 
times.

$$
\begin{aligned}
\Psi_{l, m, n, p}^{+}(x) \sim & \sum_{j=0}^{\infty} \sum_{k=0}^{\infty} C_{l, m, n, p, j, k}^{+} r^{\frac{2 j-2 l+1}{2 \beta}+2 k} \sin \frac{(2 j-2 l+1) \theta}{2 \beta} \\
& +\sum_{j=1}^{\left[\left(l+q^{\prime}-1\right) / q\right]} \sum_{k=0}^{\infty} D_{l, m, n, p, l-1+q^{\prime}-j q, k+(2 j-1) s^{\prime}}^{+} r^{(2 j-1) s^{\prime}+2 k} \log r \\
& \times \sin (2 j-1) s^{\prime} \theta . \\
\Psi_{l, m, n, p}^{-}(x) \sim & \sum_{j=0}^{\infty} \sum_{k=0}^{\infty} C_{l, m, n, p, j, k}^{-} r^{\frac{2 j-2 m+1}{2(1-\beta)}+2 k} \sin \frac{(2 j-2 m+1)(\pi-\theta)}{2(1-\beta)} \\
& +\sum_{j=1}^{\left[\left(m+t^{\prime}-1\right) / t\right]} \sum_{k=0}^{\infty} D_{l, m, n, p, m-1+t^{\prime}-j t, k+(2 j-1) s^{\prime}}^{-} r^{(2 j-1) s^{\prime}+2 k} \log r \\
& \times \sin (2 j-1) s^{\prime}(\pi-\theta) .
\end{aligned}
$$

The function $v_{l, m, n, 0}(\xi)$ admits the following asymptotic expansions as $\rho \rightarrow \infty$ which can be differentiated term by term once.

$$
\begin{aligned}
v_{l, m, n, 0}(\xi) \sim & \delta_{m, 0} C_{l, n}^{+} \rho^{\frac{2 l-1}{2 \beta}+2 n} \sin \frac{(2 l-1) \theta}{2 \beta} \\
& +\sum_{k=0}^{\infty} \sum_{h=0}^{n} K_{l, m, n, 0, k, h}^{+} \rho^{\frac{2 l-2 k-3}{2 \beta}+2 h} \sin \frac{(2 l-2 k-3) \theta}{2 \beta} \\
& +\sum_{j=1}^{\left[\left(n+s^{\prime}\right) / s\right]} \sum_{k=0}^{n-(2 j-1) s^{\prime}} L_{l, m, n, 0, l+j q-q^{\prime}-1, k+(2 j-1) s^{\prime}}^{+} \rho^{(2 j-1) s^{\prime}+2 k} \log \rho \\
v_{l, m, n, 0}(\xi) \sim & \sum_{k=0}^{\infty} \sum_{h=0}^{n} K_{l, m, n, 0, k, h}^{-} \rho^{\frac{2 m-2 k-1}{2(1-\beta)}+2 h} \sin \frac{(2 m-2 k-1)(\pi-\theta)}{2(1-\beta)} \\
& +\sum_{j=1}^{\left[\left(n+s^{\prime}\right) / s\right] n-(2 j-1) s^{\prime}} \Lambda_{+} \cdot \\
& \sum_{k=0} L_{l, m, n, 0, m+j t-t^{\prime}, k+(2 j-1) s^{\prime}}^{-} \rho^{(2 j-1) s^{\prime}+2 k} \log \rho \\
& \times \sin (2 j-1) s^{\prime}(\pi-\theta) \quad \text { on } \Lambda_{-} .
\end{aligned}
$$

For $p \geq 1$, the function $v_{l, m, n, p}(\xi)$ admits the following asymptotic expansions as $\rho \rightarrow \infty$ which can be differentiated term by term once. 


$$
\begin{aligned}
v_{l, m, n, p}(\xi) \sim & \sum_{j=1}^{1+[n / s]} \sum_{k=0}^{n+s-j s} M_{l, m, n, p, j, k}^{+} \rho^{(2 j-1) s^{\prime}+2 k} \sin (2 j-1) s^{\prime} \theta \\
& +\sum_{k=0}^{\infty} \sum_{h=0}^{n} K_{l, m, n, p, k, h}^{+} \rho^{\frac{2 l-2 k-3}{2 \beta}+2 h} \sin \frac{(2 l-2 k-3) \theta}{2 \beta} \\
& +\sum_{j=1}^{\left[\left(n+s^{\prime}\right) / s\right]} \sum_{k=0}^{n-(2 j-1) s^{\prime}} L_{l, m, n, p, l+j q-q^{\prime}-1, k+(2 j-1) s^{\prime}}^{+} \rho^{(2 j-1) s^{\prime}+2 k} \log \rho \\
v_{l, m, n, p}(\xi) \sim & \sum_{j=1}^{1+[n / s]} \times \sin (2 j-1) s^{\prime} \theta \text { on } \Lambda_{+} \cdot \\
& +\sum_{k=0}^{\infty} \sum_{h=0}^{n} K_{l, m, n, p, k, h}^{-} \rho^{\frac{2 m-2 k-1}{2(1-\beta)}+2 h} \sin \frac{(2 m-2 k-1)(\pi-\theta)}{2(1-\beta)} \\
& +\sum_{j=1}^{\left[\left(n+s^{\prime}\right) / s\right] n-(2 j-1) s^{\prime}} \sum_{k=0}^{-} L_{l, m, n, p, m+j t-t^{\prime}, k+(2 j-1) s^{\prime}}^{-} \rho^{(2 j-1) s^{\prime}+2 k} \log \rho \\
& \times \sin (2 j-1) s^{\prime}(\pi-\theta) \quad \text { on } \Lambda_{-} \cdot
\end{aligned}
$$

(iii) The following matching conditions hold.

$$
C_{l, m, n, p, j, k}^{+}=K_{j+1, m, n+k, p, l-1, k}^{+}
$$

for $l \geq 1, m \geq 0, n \geq 0, p \geq 0, j \geq 0, k \geq 0$.

$$
D_{l, m, n, p, l-1-j q+q^{\prime}, k+(2 j-1) s^{\prime}}^{+}=L_{l-j q+q^{\prime}, m, n+k+(2 j-1) s^{\prime}, p, l-1, k+(2 j-1) s^{\prime}}^{+}
$$

for $l \geq q^{\prime}, m \geq 0, n \geq 0, p \geq 0,1 \leq j \leq\left[\left(l+q^{\prime}-1\right) / q\right], k \geq 0$.

$$
C_{l, m, n, p, j, k}^{-}=K_{l, j, n+k, p, m-1, k}^{-}
$$

for $l \geq 1, m \geq 1, n \geq 0, p \geq 0, j \geq 0, k \geq 0$.

$$
D_{l, m, n, p, m-1+t^{\prime}-j t, k+(2 j-1) s^{\prime}}^{-}=L_{l, m-1+t^{\prime}-j t, n+k+(2 j-1) s^{\prime}, p, m-1, k+(2 j-1) s^{\prime}}^{-}
$$

for $l \geq t^{\prime}, m \geq 1, n \geq 0, p \geq 0,1 \leq j \leq\left[\left(m+t^{\prime}-1\right) / t\right], k \geq 0$.

$$
L_{l, m, n, p, l+j q-q^{\prime}-1, k+(2 j-1) s^{\prime}}^{+}=-M_{l, m, n-s^{\prime}, p+1, j, k}^{+}
$$

for $l \geq 1, m \geq 0, n \geq s^{\prime}, p \geq 0,1 \leq j \leq\left[\left(n+s^{\prime}\right) / s\right], 0 \leq k \leq n-(2 j-1) s^{\prime}$.

$$
L_{l, m, n, p, m+j t-t^{\prime}, k+(2 j-1) s^{\prime}}^{-}=-M_{l, m, n-s^{\prime}, p+1, j, k}^{-}
$$

for $l \geq 1, m \geq 0, n \geq s^{\prime}, p \geq 0,1 \leq j \leq\left[\left(n+s^{\prime}\right) / s\right], 0 \leq k \leq n-(2 j-1) s^{\prime}$. 
Proof. The coefficients of (5.1)-(5.4) are constructed by an induction procedure similar to Lemma 3.2. In order to obtain the logarithmic asymptotic expansions in (ii) of this theorem, we have only to replace the formal power series solutions (1.8), (1.17), (3.9), and (3.10) in the proofs of Lemmas 1.3 and 3.2 by other formal power series solutions containing logarithmic terms. One can construct such logarithmic formal power series solutions by using the formula

$$
\begin{aligned}
& \Delta_{x}\left(r^{(2 j-1) s^{\prime}+2 k} \log r \sin (2 j-1) s^{\prime} \theta\right) \\
& =\left\{(2 j-1) s^{\prime}+2 k+1\right\} r^{(2 j-1) s^{\prime}+2 k-2} \sin (2 j-1) s^{\prime} \theta \\
& \quad+4 k\left\{(2 j-1) s^{\prime}+k\right\} r^{(2 j-1) s^{\prime}+2 k-2} \log r \sin (2 j-1) s^{\prime} \theta
\end{aligned}
$$

as well as (1.7).

\section{Remarks}

Throughout this section we assume (A.1), (A.2), and $\lambda_{1}^{+}=\lambda_{1}^{-}$. We compare Theorems 0.2 and 0.4 with our previous results [9, Theorems 0.1 and 0.2 ]:

Theorem 6.1. Assume that $\alpha=\pi / 2$. Then the eigenvalue $\lambda_{2}(\epsilon)$ admits the asymptotic expansion

$$
\lambda_{2}(\epsilon) \sim \lambda_{1}^{+}+\sum_{m=1}^{\infty} \sum_{n=0}^{m-1} \lambda_{m, n} \epsilon^{2 m}(\log \epsilon)^{n} \quad \text { as } \epsilon \rightarrow 0,
$$

where

$$
\lambda_{1,0}=\frac{\pi}{8}\left(\left(C_{1,0}^{+}\right)^{2}+\left(C_{1,0}^{-}\right)^{2}\right)>0
$$

We set

$$
K=\left\{j \geq 2 ; \quad \frac{C_{j, j}^{+}}{C_{1,1}^{+}} \neq \frac{C_{j, j}^{-}}{C_{1,1}^{-}}\right\}
$$

If $K \neq \emptyset$ then the eigenvalue $\lambda_{1}(\epsilon)$ admits the asymptotic expansion

$$
\lambda_{1}(\epsilon) \sim \lambda_{1}^{+}+\sum_{i=2 \nu-1}^{\infty} \sum_{j=0}^{[(i-2 \nu+1) / 3]} \mu_{i, j} \epsilon^{2 i}(\log \epsilon)^{j} \quad \text { as } \epsilon \rightarrow 0, \quad \mu_{2 \nu-1,0}>0
$$

where $\nu=\min K$. If $K=\emptyset$ then $\lambda_{1}(\epsilon)=\lambda_{1}^{+}$for sufficiently small $\epsilon>0$.

Theorem 6.2. Assume that $\alpha=\pi / 2$. We also suppose that there exists $\tau \in C^{\infty}\left(\left[0, t_{0}\right]\right)$ such that $\gamma(t)=(\tau(t), t)$ on $\left[0, t_{0}\right]$ and $\tau\left(t_{0}\right)=0$.

Then $K=\emptyset$ if and only if $\Omega$ is symmetric with respect to $x_{2}$-axis and $\tau=0$ on $\left[0, t_{0}\right]$. 
Combining the above two theorems with Theorems 0.2 and 0.4 , we arrive at the following implications. For all $\alpha \in(0, \pi)$, the order of the leading term of the asymptotic expansion of $\lambda_{2}(\epsilon)$ is $\min \left\{\frac{1}{1-\beta}, \frac{1}{\beta}\right\}$. In the case when $\alpha \in(0, \pi / 2) \cup$ $(\pi / 2, \pi)$, the order of the leading term of the asymptotic expansion of $\lambda_{1}(\epsilon)$ is $\max \left\{\frac{1}{1-\beta}, \frac{1}{\beta}\right\}$. However, in the case when $\alpha=\pi / 2$, the order of the leading term of the asymptotic expansion of $\lambda_{1}(\epsilon)$ depends on the set $K$ which reflects the geometric relation between $\Omega_{+}$and $\Omega_{-}$.

Acknowledgement. The author thanks the referee for useful comments which improved the paper.

\title{
References
}

[1] M. Dauge, Elliptic boundary value problems on coner domains, Lecture Note in Math., Vol. 1341, Springer-Verlag, 1988.

[2] M. Dauge and B. Helffer, Eigenvalues variation. II. Multidimensional problems, J. Differential Equations, 104 (1993), 263-297.

[ 3 ] R. Gadyl'shin and A. Il'in, Asymptotic behavior of the eigenvalues of the Dirichlet problem in a domain with a narrow slit, Sbornik Math., 189 (1998), 503-526.

[4] D. Gilbarg and N.S. Trudinger, Elliptic partial differential equations of second order, Springer-Verlag, 1998.

[5] A. Il'in, Matching of asymptotic expansions of solutions of boundary value problems, Translations of Mathematical Monographs, Vol. 102, American Mathematical Society, 1992.

[6] V.A. Kondrat'ev, Boundary problems for elliptic equations in domains with conical or angular points, Trans. Moscow Math. Soc., 16 (1967), 227-313.

[ 7 ] S. Nazarov and B. Plamenevsky, Elliptic problems on domains with piecewise smooth boundaries, de Gruyter Expositions in Mathematics, Vol. 13, Walter de Gruyter, 1994.

[8] Z. Nehari, Conformal mapping, Dover, 1952.

[9] K. Yoshitomi, Eigenvalue problems on domains with cracks, preprint.

[10] K. Yoshitomi, Band spectrum of the Laplacian on a slab with the Dirichlet boundary condition on a grid, Kyushu J. Math., 57 (2003), 87-116.

\author{
Department of Mathematics \\ Tokyo Metropolitan University \\ Minami-OhsaWa 1-1, HaChIOJI-ShI \\ TOKYO 192-0397, JAPAN \\ E-mail: yositomi@comp.metro-u.ac.jp
}

